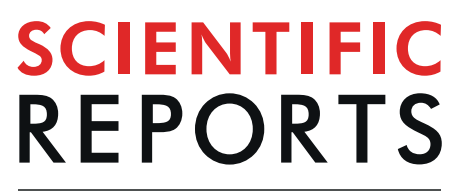

natureresearch

Check for updates

\title{
OPEN RNA-seq analysis reveals TRPC genes to impact an unexpected number of metabolic and regulatory pathways
}

\author{
Karina Formoso ${ }^{1,4}$, Sebastian Susperreguy ${ }^{1}$, Marc Freichel ${ }^{2}$ \& Lutz Birnbaumer ${ }^{1,3 凶}$
}

The seven-member transient receptor potential canonical genes (TRPC1-7) encode cation channels linked to several human diseases. There is little understanding of the participation of each TRPC in each pathology, considering functional redundancy. Also, most of the inhibitors available are not specific. Thus, we developed mice that lack all of the TRPCs and performed a transcriptome analysis in eight tissues. The aim of this research was to address the impact of the absence of all TRPC channels on gene expression. We obtained a total of 4305 differentially expressed genes (DEGs) in at least one tissue where spleen showed the highest number of DEGs (1371). Just 21 genes were modified in all the tissues. Performing a pathway enrichment analysis, we found that many important signaling pathways were modified in more than one tissue, including PI3K (phosphatidylinositol 3-kinase/protein kinase-B) signaling pathway, cytokine-cytokine receptor interaction, extracellular matrix (ECM)-receptor interaction and circadian rhythms. We describe for the first time the changes at the transcriptome level due to the lack of all TRPC proteins in a mouse model and provide a starting point to understand the function of TRPC channels and their possible roles in pathologies.

The transient receptor potential canonical (TRPC) are non-specific cation channels and the first group to be described among the mammalian TRP family. The TRPC family is composed of seven different proteins (TRPC1-7) encoded in seven different genes, though TRPC2 is a pseudogene in humans ${ }^{1}$. All seven channels share structural features. All of them possess six transmembrane helices, a hydrophobic loop contributing to the outer vestibule and upper gate of the pore, several, three to four ankyrin repeats, coiled-coil domains in the N-and C-termini and, a C-terminal proline-rich region ${ }^{2,3}$. In spite of recent advances in elucidating the atomic structure of TRPCs their physiologic functions are still understood only poorly ${ }^{4-6}$. Based on sequence similarity, the 7 TRPC proteins segregate into 4 subgroups: TRPC1, TRPC4 \& 5, TRPC3, 6 \& 7 and TRPC2. TRPCs were originally proposed to be activated by store depletion, which may not be so, but, most if not all, TRPCs appear to be activated by diacylglycerol $(\mathrm{DAG})^{7,8}$. In addition to allowing passage of $\mathrm{Na}+$ and $\mathrm{K}+$, TRPC channels allow passage of Calcium. TRPC proteins assemble into cation channels as homo- and hetero-tetramers including 2 or 3 TRPCs subtypes ${ }^{9-11}$. TRPC channels have been linked to various human diseases that show a dysregulation of store-operated Calcium entry (SOCE), such as in cancer ${ }^{12}$, cardiovascular $^{13}$, pulmonary $^{14}$, neuropsychiatric and neurological diseases ${ }^{15}$. Also, TRPC channels have been repeatedly implicated in NFAT activation, particularly in cardiac tissue pathologies $^{16}$. Thus transcriptional regulation by NFAT and/or other transcription factors might be the basis of numerous phenotypes developed in KO mice that resulted from dysregulated expression of genes.

Despite the great increase in the last decades in the number of publications in the TRPC field, the exact function, and mechanism of action of TRPCs have not been fully described. Regarding their role in different pathologies, there is little understanding of the degree to which each TRPC participates in each pathology, considering that they present functional redundancy. Also, it is important to highlight that the inhibitors available for TRPCs are not specific, most of the drugs affect more than one TRPC and in many cases other proteins such as ORAI1, so

${ }^{1}$ Institute for Biomedical Research (BIOMED UCA-CONICET). School of Medical Sciences, Catholic University of Argentina (UCA), Buenos Aires, C1107AFF, Argentina. ${ }^{2}$ Institute of Physiology and Pathophysiology, Heidelberg University, 69120, Heidelberg, Germany. ${ }^{3}$ Neurobiology Laboratory, National Institute of Environmental Health Sciences (NIEHS), Research Triangle Park, North Carolina, 27709, USA. ${ }^{4}$ Karina Formoso is a postdoctoral research fellow of the National Scientific and Technical Research Council (CONICET). 『email: birnbau1@gmail.com 


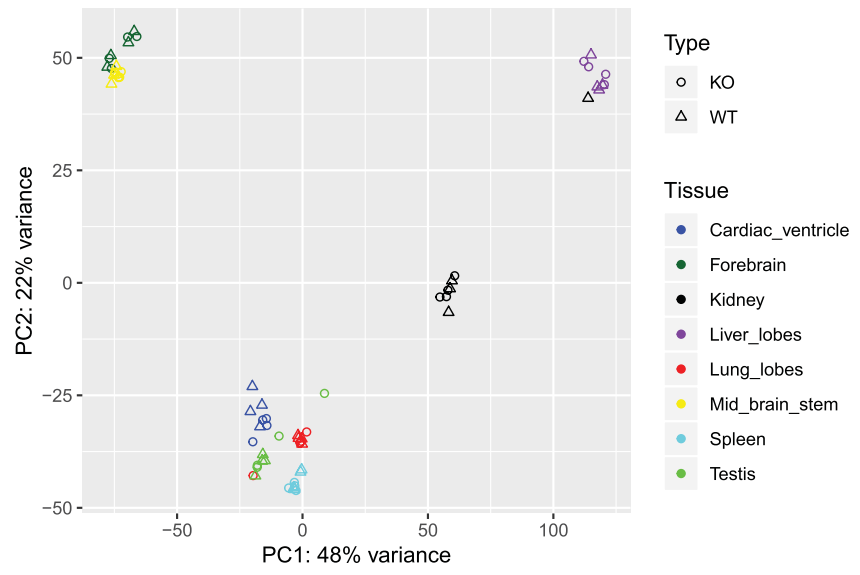

Figure 1. Principal component analysis (PCA) of WT and TRPC heptaKO mouse tissues. PCA plot depicts the distribution of the gene profile of each sample, KO and WT, and each tissue.

a thorough study of TRPCs is challenging. As a consequence of the complex scenario found in the field of TRPC research, we developed mice that lack all of the TRPC channels (TRPC hepta knockout mice) that have been experimentally characterized (see refs. ${ }^{17,18}$ ). The aim of this work was to address the impact of the absence of all TRPC channels on gene expression in several tissues simultaneously and ask at a global level which functions depend on TRPC channels during their normal functioning.

\section{Results}

In order to determine the relevance of TRPCs expression in different tissues four TRPC heptaKO and four WT three-month-old male mice were used to obtain RNA samples from Cardiac ventricle (Heart), Kidney, Spleen, Testis, Liver lobes (Liver), Brain (Midbrain and Forebrain), and Lung lobes (Lung). From these samples, we obtained $1.95 \times 10^{9} 51$-nt reads, of which $1.67 \times 10^{9}(85 \%)$ mapped uniquely to the mouse genome for an average of 26.07 million 51-nt reads per sample, using Illumina Bioanalyzer technology. We found a good identification of the sequence throughout the readings and the quality score per sequence obtained was high (near 40). Overall the data had an appropriate quality to continue the analysis.

To visualize the variation in expression between samples, we performed a Principal Component Analysis (PCA) where we determined that the principal variation among samples is, as expected, due to the tissue and not to the sample type, eg, WT vs KO (Fig. 1). When the samples were analyzed by tissue, they were segregated by KO or WT phenotype, except for three of the 64 samples (1 WT kidney, 1 KO lung, and 1 KO testis) which were discarded for subsequent analyses (Fig. 2). Our RNAseq analysis allowed us to determine that the number of genes expressed per tissue was between 11.4 and 15.6 thousand.

To determine which genes change their expression between $\mathrm{KO}$ and WT, we used edgeR which is an efficient program for determining the most significant Differentially Expressed Genes (DEGs). As a unifying criterion, an adjusted p-value of 0.01 or less and a minimum fold change of $50 \%$ was used. The highest differences in the number of DEGs among the tissues were found both in the liver and the spleen (Fig. 3A). A total of 4305 genes were found to be differentially expressed in at least one tissue. From these 77\% (3312) are tissue-specific. A heat map that includes these 3312 genes (Fig. 3B), shows both the variability between tissues and also between mice of the WT and KO genotypes. A complete list of differentially expressed genes can be found in Supplementary Table 1.

To analyze the interaction between the identified common targets, a protein-protein interaction (PPI) network was constructed for each tissue using STRING. STRING is a database of known and predicted protein-protein interactions that allows searching in a list of possibly interacting genes or proteins ${ }^{19}$. Next, the network was retrieved and was further analyzed using the Cytoscape software. Cytoscape is an open-source bioinformatics software platform that allows us to visualize and analyze molecular interaction networks. From these analyses, we obtained the top hub proteins, meaning the ones with the highest degree of connectivity, which represents the number of interactions associated with the protein. After that, we used the molecular complex detection plug-in (MCODE) to identify modules, i.e. densely connected nodes. Each module may but need not represent molecular complexes. This is a first approach to determine the possible functions of the proteins affected by the absence of TRPC channels. Finally, we performed a functional enrichment analysis. The aim of this type of analysis was to identify biological issues or functions that represent the differential signals detected. This is carried out through different strategies. The first, over-representation analysis (ORA) seeks to identify which biological pathways are over-represented in the list of DEGs. The second strategy, the network-based gene set enrichment analysis (NGSEA) seeks to identify coordinated signals from genes associated with a given biological subject compatible with interaction networks independently surveyed (eg: metabolic pathway, signaling, gene regulation networks). For each tissue, we report four analyses, one based on Gene Ontology (GO) and three based on the Kyoto Encyclopedia of Genes and Genomes (KEGG) pathways. The ORA analysis was performed both for GO and KEGG. The NGSEA analysis was performed with the Gene Graph Enrichment Analysis (GGEA) and the Signaling Pathway Impact Analysis. (SPIA) both with KEGG pathways. GGEA is a method that allows the search of enrichment in a group of genes, taking into account the inhibitory and excitatory nature between interactions 

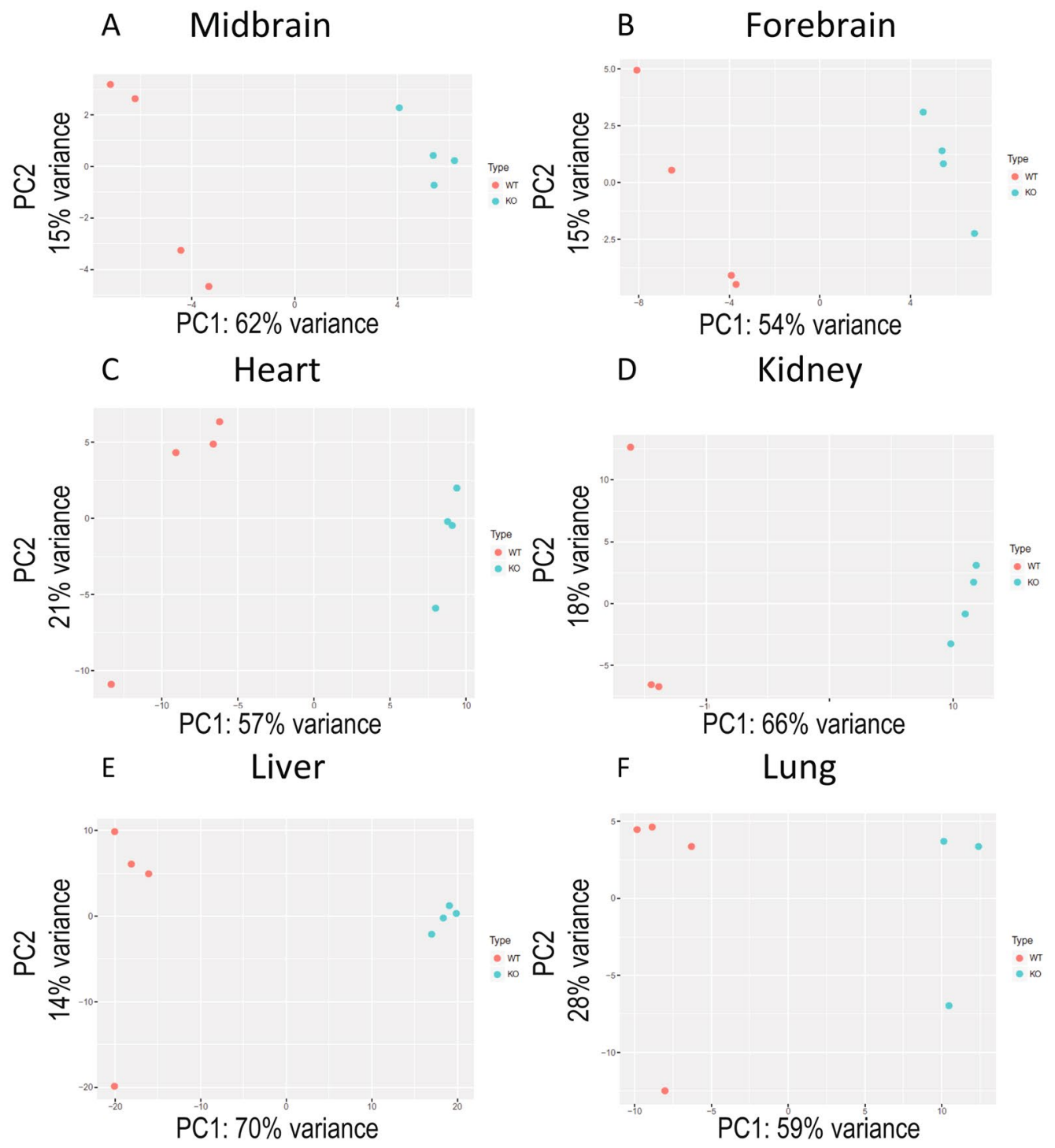

G

Spleen

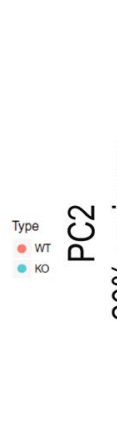

H Testis
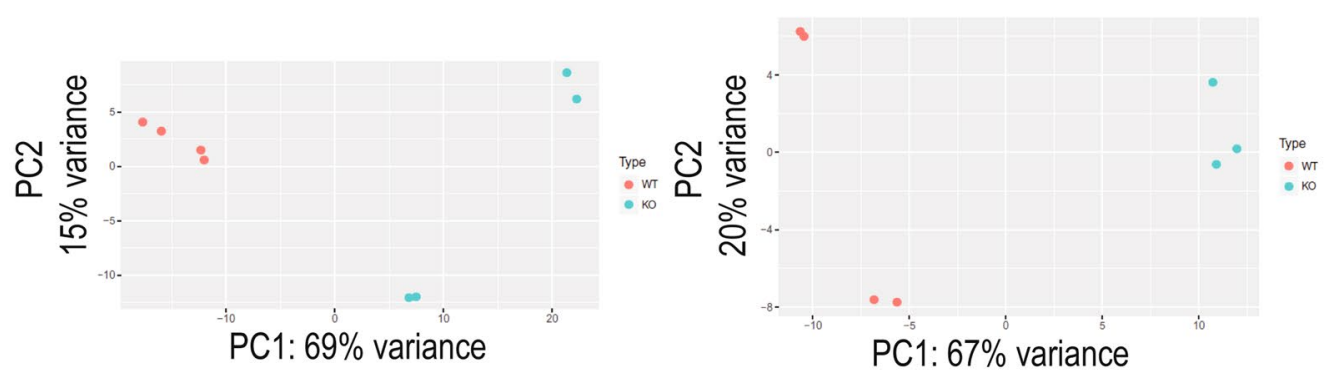

Figure 2. PCA analyses of each sample.

that may exist between them. SPIA analyses whether the expression changes detected are propagated in a manner compatible with the topology of the pathway interactions in question. This analysis allowed us to obtain a list of affected pathways with a false discovery rate (FDR) $<0.05$ for each tissue. Table 1 (Metabolic and signaling pathways impacted by the loss of TRPC expression).

Analysis of cardiac ventricle reads. In the heart, TRPC channel expression is low and is mostly localized to the peripheral plasma membrane in adult cardiac myocytes, though the expression of TRPC channels is increased in cardiac hypertrophy and heart failure. Studies in KO mice showed that they are protected from cardiac hypertrophy and that TRPCs might be contributing to cardiac hypertrophy through the Calcineurin-NFAT 

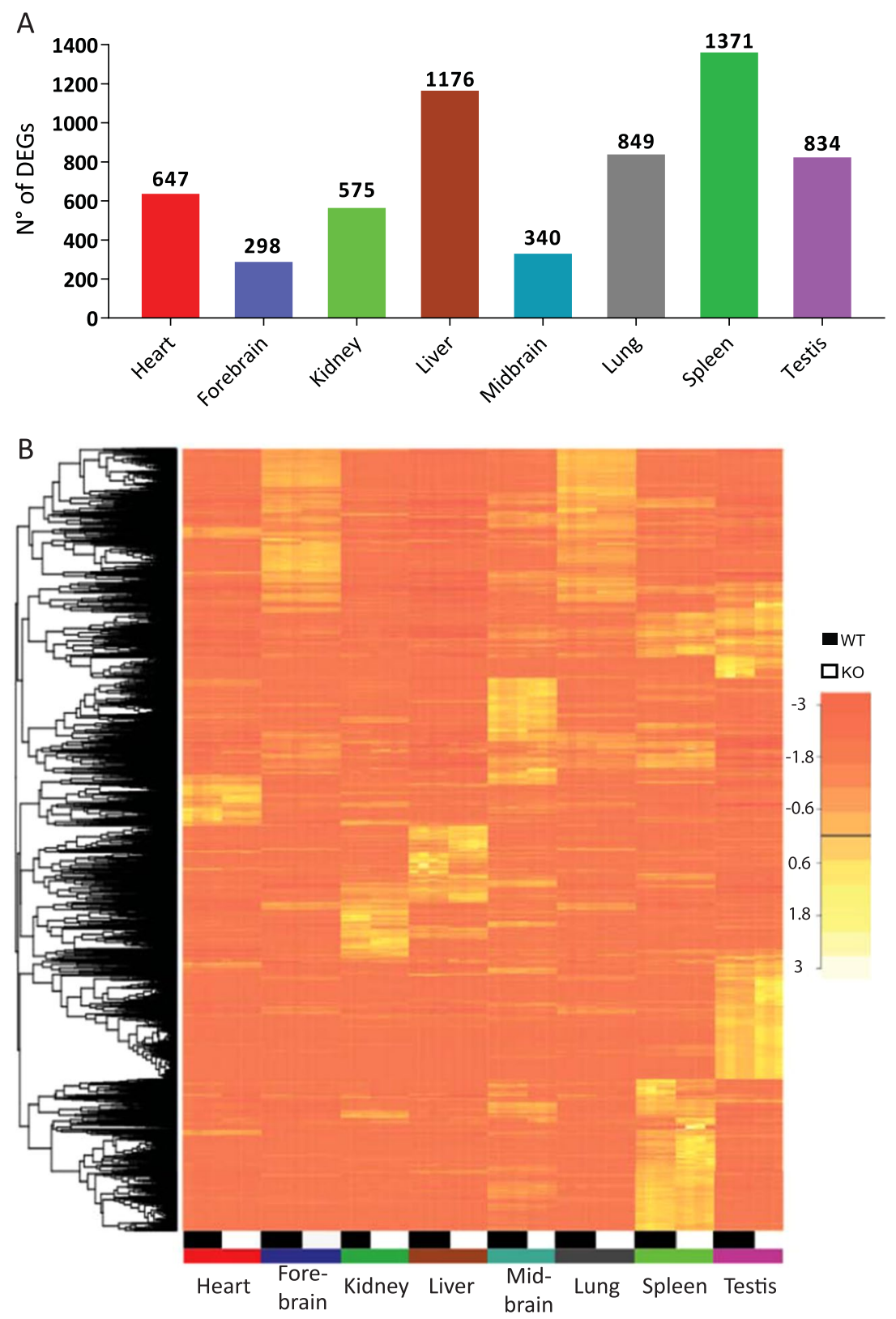

Figure 3. Differentially expressed genes in the KO mice. (A) Number of differentially expressed genes (DEGs) per tissue. Bars represent the number of statistically DEGs at $\mathrm{p}<0.01$ for a $50 \%$ change in each tissue. (B) Genes with statistically significant alterations in at least one tissue are depicted in a heat map. Each column depicts changes in a WT and a heptaKO and each row a gene.

pathway ${ }^{16}$. Research from our laboratory established that the absence of TRPC 3 and 6 protects mouse hearts in an Ischemia-reperfusion injury model ${ }^{16}$. From the present analysis, we determined that there are 490 protein-coding DEGs with an adjusted $\mathrm{p}$-value $<0.01$ and a fold change of at least $50 \%$. The PPI network was retrieved and consisted of 482 nodes and 754 edges with a p-value of 1.0e-16 (Fig. 4A). The top hub proteins were selected from the network with a degree $>13$ (Fig. 4C). Among them, we found the matrix metallopeptidase 9 (mmp9). The top 4 significant modules were selected and are shown in Fig. 4D (I-IV). The module I proteins are related to muscle contraction, module II proteins are related to ribosome structure, module III are proteins related to circadian rhythm and finally, module IV are proteins related to antigen processing and presentation. The most affected pathway in the heart was circadian rhythm, in agreement with the PPI network analysis and the role of TRPC6 and 7 seen in double $\mathrm{KO}$ mice $^{20}$. Also, Ras, Rap1, PLD signaling and, antigen processing and presentation pathways were significantly affected by the deletion of the TRPC genes (Fig. 4B).

Analysis of central nervous system reads. With respect to the nervous system, the expression of TRPCs is ubiquitous and is regulated in different developmental stages ${ }^{15,21}$. Also, TRPCs have been related to several pathologies of the nervous system ${ }^{15,22}$ many related to diverse functions such as neuronal firing, neurite extension 


\begin{tabular}{|l|l|l|l|l|l|l|l|}
\hline & Heart & Brain & Lung & Liver & Spleen & Kidney & Testis \\
\hline Antigen processing and presentation & $\mathrm{X}$ & & $\mathrm{X}$ & & & $\mathrm{X}$ & \\
\hline Cell Adhesion & & & $\mathrm{X}$ & & $\mathrm{X}$ & $\mathrm{X}$ & \\
\hline Circadian regulation of Gene expression & $\mathrm{X}$ & & & & & $\mathrm{X}$ & \\
\hline Circadian Rhythm & $\mathrm{X}$ & & $\mathrm{X}$ & & & $\mathrm{X}$ & \\
\hline Cytokine-cytokine receptor interaction & & $\mathrm{X}$ & $\mathrm{X}$ & & $\mathrm{X}$ & & $\mathrm{X}$ \\
\hline ECM-receptor interaction & & $\mathrm{X}$ & $\mathrm{X}$ & $\mathrm{X}$ & $\mathrm{X}$ & $\mathrm{X}$ & $\mathrm{X}$ \\
\hline Focal adhesion & & $\mathrm{X}$ & & & $\mathrm{X}$ & $\mathrm{X}$ & $\mathrm{X}$ \\
\hline Phospholipase D signaling pathway & $\mathrm{X}$ & & & & & & $\mathrm{X}$ \\
\hline PI3K-Akt signaling pathway & $\mathrm{X}$ & $\mathrm{X}$ & $\mathrm{X}$ & & & $\mathrm{X}$ & $\mathrm{X}$ \\
\hline positive regulation of ERK1 and ERK2 cascade & & & $\mathrm{X}$ & & $\mathrm{X}$ & & \\
\hline PPAR signaling pathway & & & $\mathrm{X}$ & $\mathrm{X}$ & & $\mathrm{X}$ & \\
\hline Protein digestion and absorption & & & $\mathrm{X}$ & & $\mathrm{X}$ & $\mathrm{X}$ & \\
\hline Rap1 signaling pathway & $\mathrm{X}$ & & & & $\mathrm{X}$ & & \\
\hline Ras signaling pathway & $\mathrm{X}$ & & & & & & $\mathrm{X}$ \\
\hline
\end{tabular}

Table 1. Pathways affected in 2 or more of the tissues analyzed.

and growth cone guidance ${ }^{21}$. We found 185 protein-coding DEGs in the forebrain and 210 DEGs in the midbrain which included basal ganglia and cerebellum. The PPI network retrieved from the forebrain protein-coding DEGs consisted of 180 nodes and 116 edges with a p-value of 0.33 , meaning it is not statistically significant, for midbrain, it consisted of 216 nodes, 209 edges and a p-value, of $2.77 \mathrm{e}-5$ (Figs. 5A and 6A, respectively). The top hub proteins were selected from the network (Figs. 5C and 6C). Using Cytoscape and MCODE we obtained 1 module for forebrain and 3 for midbrain (Figs. 5D and 6D) where we found in both a module for ribosomal proteins (5D.I and 6D.I). For midbrain, we found also a module for Stress response proteins (6D.II) and cell cycle proteins (6D.III). From the enrichment analyses, we found that though the modified signaling pathways are few they tend to repeat between forebrain and midbrain, leading to the idea of a conserved functionality of TRPCs throughout the brain (Figs. 5B and 6B). Among them, we found modified focal adhesions, ECM receptor interaction, cytokine-cytokine receptor interaction and, manganese ion transport in both forebrain and midbrain. In forebrain we also found PI3K-Akt and hippo signaling pathway.

Analysis of kidney reads. In the kidney, TRPCs $1,3,4,5$ and 6 are predominantly expressed in the glomerulus, particularly, in mesangial cells and podocytes ${ }^{23-28}$. Here we found that the kidney showed 433 protein-coding DEGs. The PPI network analyzed with STRING consisted of 430 nodes and 812 edges with a p-value of 1.0e-16 (Fig. 7A). The top hub genes were selected from the network (Fig. 7C). The top 4 significant modules were selected and are shown in Fig. 7D. Module I proteins are related to regulation of lipolysis, Module II is composed mostly of ribosomal proteins and proteins related to PI3K-Akt signaling pathway, Module III has proteins related to circadian rhythm and module IV shows proteins related to basement membrane components. From the pathway enrichment analysis we found ECM-receptor interactions, circadian rhythm, the PI3K signaling pathway similar to the results obtained for heart, and brain, Also, we found focal adhesions, protein digestion and absorption, and antigen processing and presentation. Finding repeated pathways in different tissues gave us the first clue that there are common pathways affected throughout the animal (Fig. 7B).

Analysis of lung lobe reads. All TRPC isoforms have been detected in the pulmonary vasculature with the highest expression levels for TRPC1 and TRPC6 and lower levels for TRPC3, TRPC 4, TRPC5 and TRPC7 29-32. Moreover, the expression of TRPC1, TRPC4, and TRPC6 is higher in the proximal pulmonary arteries (which are more sensitive to hypoxia) compared to the distal pulmonary arteries ${ }^{33}$. Lung reads mapped to 676 protein-coding DEGs. The PPI network generated by STRING consisted of 668 nodes and 2945 edges with a p-value of 1.0 e-16 (Fig. 8A). The top hub genes regarding degree were selected from the network (Fig. 8C). Using the Cytoscape software and MCODE plug-in the top 4 significant modules were selected and are shown in Fig. 8D. We found a module related to cytokine-cytokine receptor interaction (8D.I), one related to extracellular matrix and basement membrane composition (8D.II), a third module is related to natural killer cell-mediated cytotoxicity (8D.III) and the last module is related to B-Cell receptor signaling (8D.IV). From the pathway enrichment analyses, we found the ECM-receptor signaling pathway, circadian rhythm, PI3K-Akt signaling pathway, cytokine-cytokine receptor interaction, cell adhesion, and migration, and pathways related to phospholipase D (PLD) signaling to be affected by the loss of TRPCs (Fig. 8B).

Analysis of testis reads. In the testis, TRPCs reported to be expressed in mammalian spermatozoa include TRPC1, TRPC3, TRPC4, TRPC6, and TRPC $2^{34}$. TRPC1 and TRPC2 have been reported to be present in the acrosomal domain of the mouse sperm head ${ }^{35,36}$. In mouse sperm, TRPC3 has been localized in the post acrosomal head and tail ${ }^{37}$ but in human spermatozoa, TRPC3 is localized on the acrosomal region and mid-piece and, on the plasma membrane of the sperm head and flagella ${ }^{38}$. TRPC4 has not been detected at the protein level in mice but in human spermatozoa, a weak TRPC4 signal was obtained in the head and a strong signal on midpiece and tail ${ }^{38}$. In mouse sperm TRPC6 has been localized in the post-acrosomal head region and sperm flagellum ${ }^{37}$ and in human sperm, TRPC6 has been localized in the mid-piece and the sperm tail ${ }^{38}$. We found 511 
A

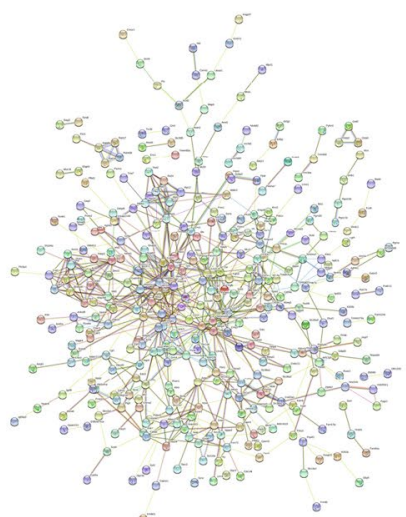

C

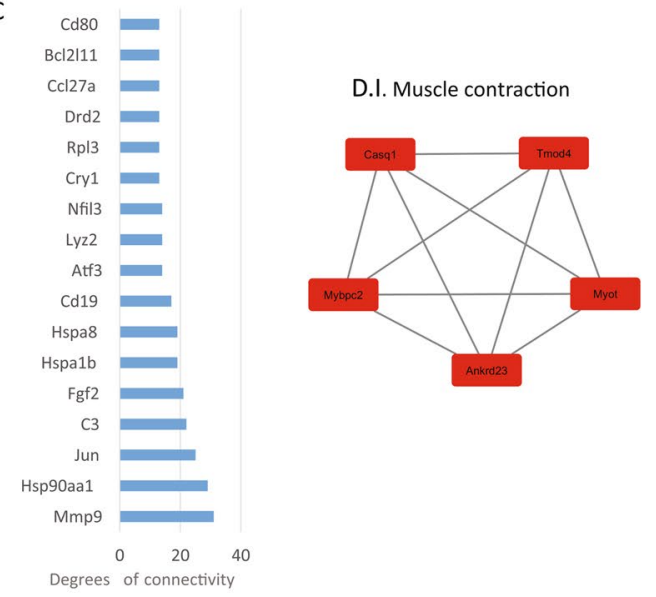

D.II. Ribosome make-up

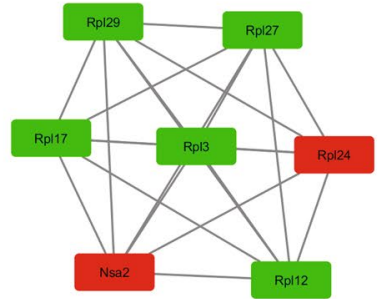

B
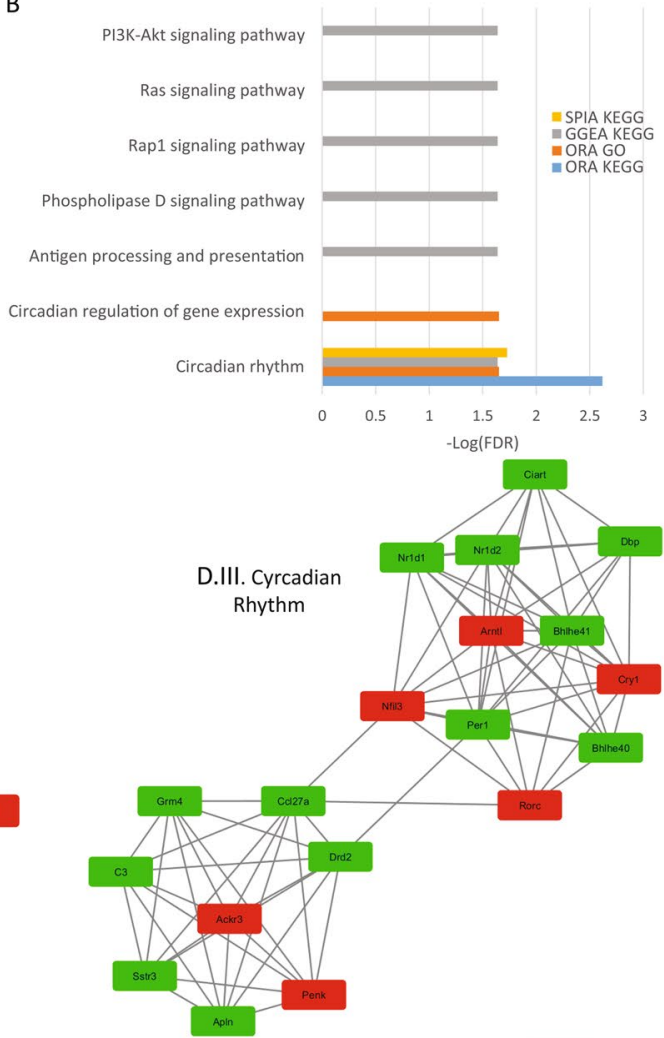

III. Cyrcadian Rhythm

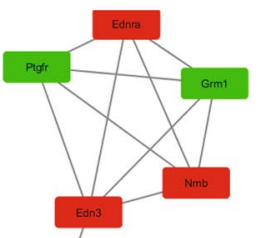

D.IV. Antigen processing and presentation
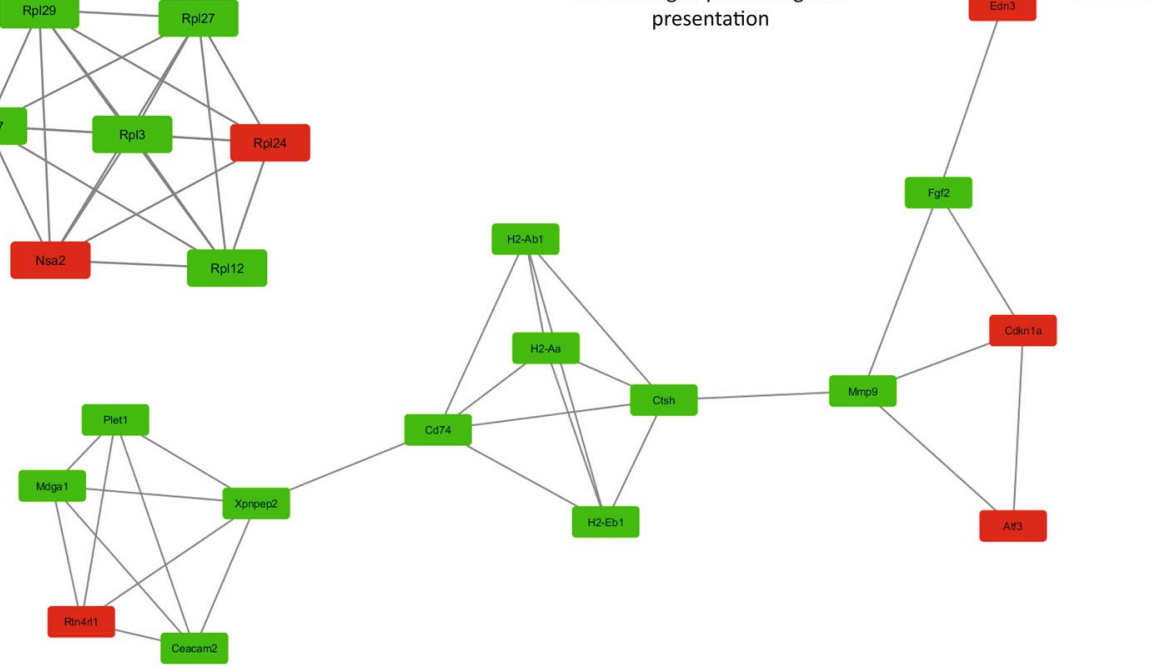

Figure 4. Heart analysis. (A) Protein-protein interaction (PPI) network generated with STRING from DEGs in the heart. The network is composed of 482 nodes and connected with 754 edges ( $p$-value of $1.0 \mathrm{e}-16$ ). The edges might be drawn in seven different colors indicating different type of evidence (Red line - fusion evidence; Green line - neighborhood evidence; Blue line - cooccurrence evidence; Purple line - experimental evidence; Yellow line - textmining evidence; Light blue line - database evidence; Black line - coexpression evidence). See also high resolution panel A in Supplementary information 2. (B) The graph shows the results of the pathway enrichment analyses for the DEGs obtained from the heart. The bars depict the negative logarithm of the false discovery rate (FDR). (C) Genes with the highest degree of connectivity obtained from the PPI network with Cytoscape. D.IIV) Top modules obtained from the PPI network using Cytoscape's plugin MCODE (Red nodes: Upregulated in hepta KO mice; Green nodes: Downregulated in heptaKO mice). 
A<smiles></smiles>

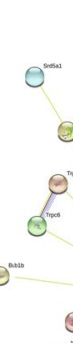

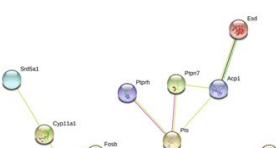

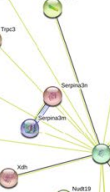<smiles>[C-]1C2CC12</smiles>

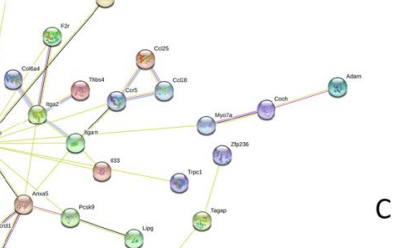

B

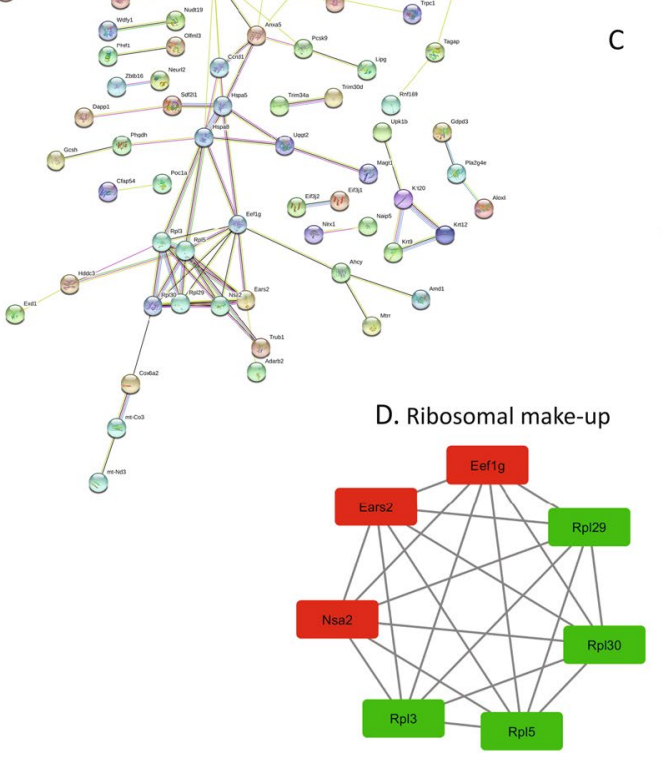

\section{(}

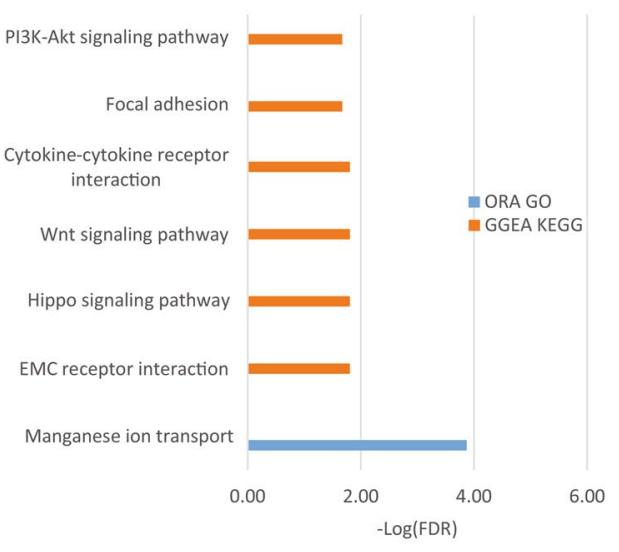

Figure 5. Forebrain analysis. (A) PPI network generated with STRING from DEGs in the forebrain. The network comprises 180 nodes and connected with 116 edges (p-value of 0.33 ). For the rest of the details see Fig. 3 and also high resolution panel A in Supplementary information 2. (B) The graph shows the results from the pathway enrichment analyses for the DEGs obtained from the Forebrain. The bars depict the negative logarithm of the false discovery rate (FDR). (C) Genes with the highest degree of connectivity obtained from the PPI network with Cytoscape. (D) Module obtained from the PPI network using Cytoscape's plugin MCODE (Red nodes: Upregulated in hepta KO mice; Green nodes: Downregulated in heptaKO mice).

protein-coding DEGs that defined a PPI network consisting of 483 nodes and 582 edges with a p-value of $4.03 \mathrm{e}$ -5 (Fig. 9A). The top hub genes regarding degree were selected from the network (Fig. 9C). The top 4 significant modules were selected and are shown in Fig. 9D. Module I has ribosome related proteins, module II is related to cholesterol metabolism, module III is formed by chromatin related proteins, and module IV has proteins related to the PLD signaling pathway. In the pathway enrichment analysis, we only obtained significant differences using GGEA analysis as shown in Fig. 9B which identified ECM-receptor interaction, Focal adhesions, PI3K-Akt, Ras, PLD signaling, Cholesterol metabolism and, cytokine-cytokine receptor interaction to be affected by the loss of TRPCs.

Analysis of the liver lobe reads. In the Liver, TRPC1, TRPC3, TRPC5, and TRPC6 were shown to be expressed $^{22,39}$. From our analysis, 554 protein-coding DEGs were found and the STRING-generated PPI network was formed by 937 nodes and 2691 edges with a p-value of $1.0 \mathrm{e}-16$ (Fig. 10A). The top hub genes are shown in Fig. 10C and the top 4 significant modules are shown in Fig. 10D where module I is formed by proteins related to ECM components, module II proteins related to regulation of lipolysis, module III is related to circadian rhythm and, finally, module IV is formed by proteins related to retinol metabolism. From the pathway enrichment analysis, we found the PPAR signaling pathway, ECM-receptor interaction, and lipid metabolic processes to be affected by the loss of TRPC expression (Fig. 10B).

Analysis of spleen reads. The bibliography regarding TRPCs in the spleen is limited, but there are publications that propose that all TRPCs are expressed in this tissue ${ }^{39}$. From the 1027 protein-coding DEGs found in this tissue, we obtained a PPI network formed by 1015 nodes and 14204 edges with a p-value of $1.0 \mathrm{e}-16$ (Fig. 11A). The top hub genes regarding degree are shown (Fig. 11C) and the top 4 significant modules are shown in Fig. 11D. Here we found modules related to protein digestion (11D.I), ribosome components (11D.II), cytokines (11D. III) and, cell cycle and DNA replication (11D.IV). The pathway enrichment analysis identified several affected pathways, among them ECM-receptor interaction, Focal adhesions, cytokine-cytokine receptor interaction and, protein digestion and absorption (Fig. 11B).

We looked for a general modification in all the tissues but the genes modified in 6 and 7 tissues were too few to give a general idea of the processes affected in the whole KO mice. Figure 12A depicts the distribution of DEGs 
A
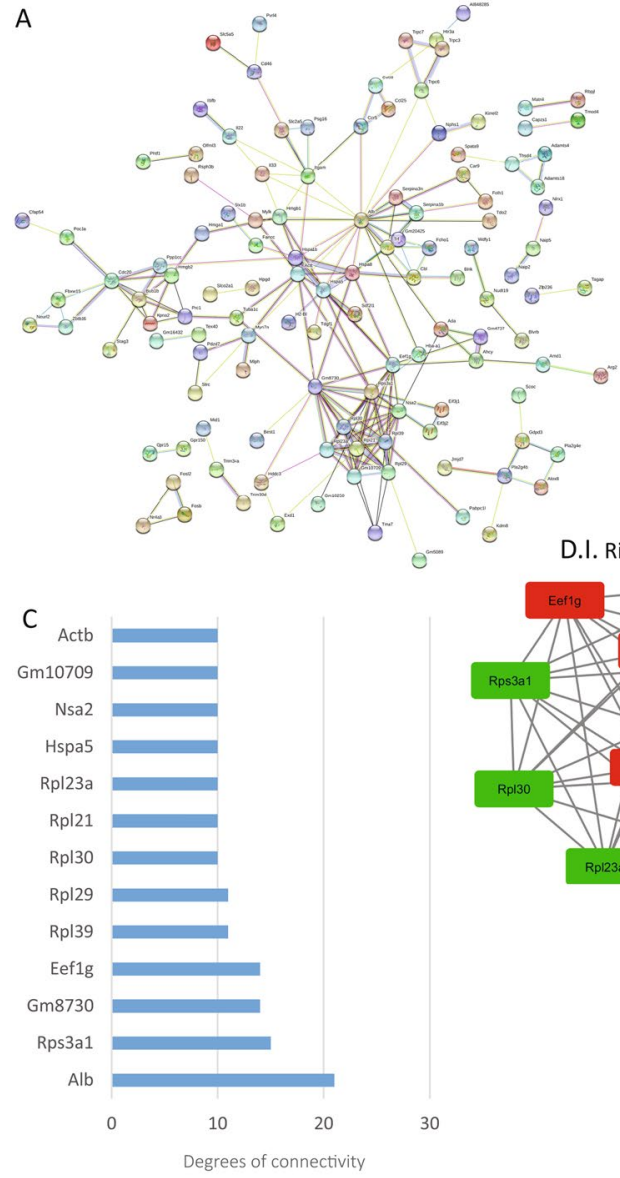

B

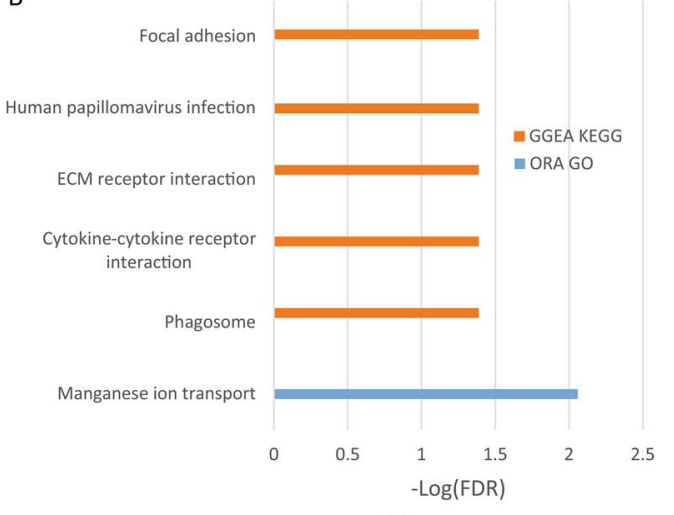

D.II. Stress response proteins
$\mathrm{C}$

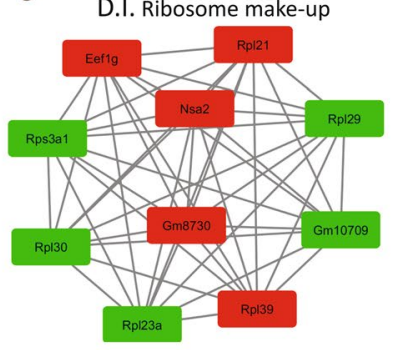

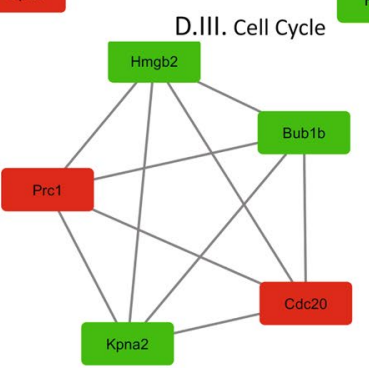

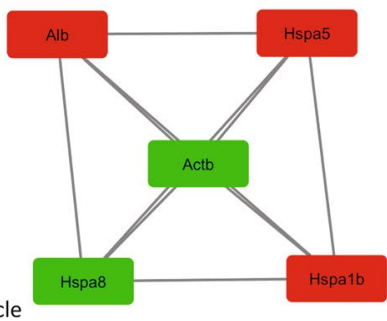

Figure 6. Midbrain (plus brain stem \& cerebellum) analysis. (A) PPI network generated with STRING from DEGs in the midbrain. The network comprises 216 nodes connected by 209 edges (p-value of 2.77 e-5). For the rest of the details see Fig. 3 and also high resolution panel A in Supplementary information 2. (B) The graph shows the results from the pathway enrichment analyses for the DEGs obtained from the Midbrain. The bars depict the negative logarithm of the false discovery rate (FDR). C) Genes with the highest degree of connectivity obtained from the PPI network with Cytoscape. D.I-III) Top three modules obtained from the PPI network using Cytoscape's plugin MCODE (Red nodes: Upregulated in hepta KO mice; Green nodes: Downregulated in heptaKO mice).

according to the number of tissues involved where we can see that only 21 genes were found to be differentially expressed in all the tissues. From the 40 genes that are differentially expressed in 7 or 8 tissues, we filtered the pseudogenes (Table 2) and analyzed the remaining genes with STRING. Regrettably, we could not find significant interactions among the 21 uniquely differentially expressed genes. Considering this, the pathway enrichment analyses allowed us to search for common pathways affected in different tissues even though the genes modified were not necessarily the same between one and the other. Once each tissue had been analyzed we determined which pathways were represented in the tissues. From this, we determined that the ECM-receptor interaction is affected in most of the tissues. Next is the PI3K signaling pathway, followed by cytokine-cytokine receptor interaction and, focal adhesion (Table 1 and Fig. 12B).

\section{Discussion}

TRPCs have been repeatedly connected to various pathologies ${ }^{15,22}$. For example, TRPC1 has been shown to maintain calcium homeostasis in a mouse model of Parkinson's disease thus limiting neuronal degeneration. Also, some symptoms are decreased when TRPC1 is expressed in neuronal cells or in vivo ${ }^{40}$. A mouse model of Rett syndrome (Mecp2 mutant mice) shows a loss of function of TRPC 3 in hippocampus neurons that leads to sensory and motor abnormalities ${ }^{41}$. Increased TRPC6 promoter activity and TRPC6 expression have been linked to the development of idiopathic pulmonary arterial hypertension, which is caused by excessive proliferation of pulmonary artery smooth muscle cells. To date there are several mutations of TRPC6 described to be responsible for the familial forms of focal segmental glomerulosclerosis in $\operatorname{man}^{42-44}$. Studies in KO mice showed that they are protected from cardiac hypertrophy and that TRPCs might be contributing to cardiac hypertrophy through Calcineurin-NFAT pathways ${ }^{45-47}$. As mentioned earlier, we reported that the absence of TRPC 3 and 6 protects mouse hearts in an Ischemia-reperfusion injury model ${ }^{16}$. 
A

B
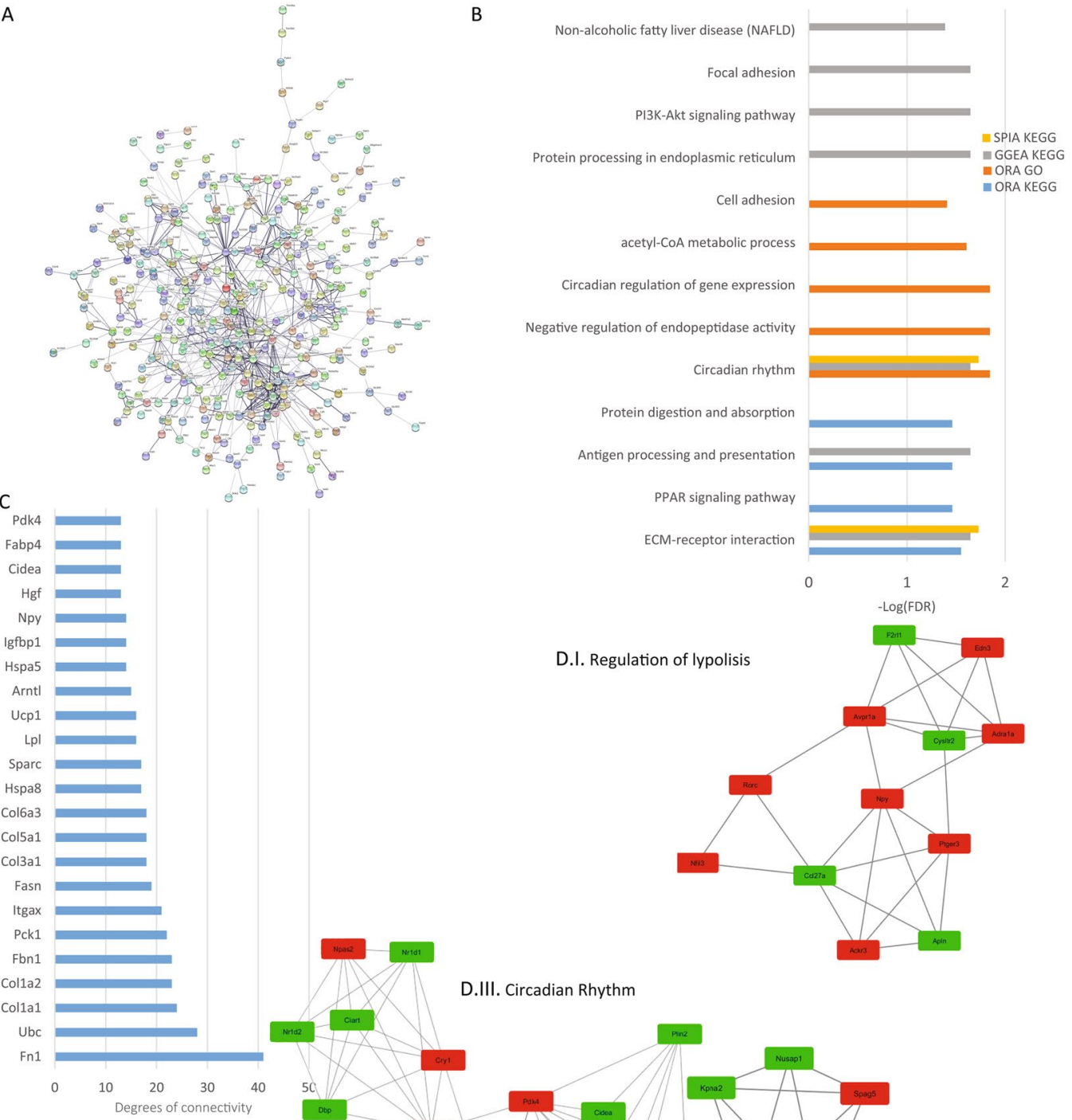

D.IV. Basement membrane components
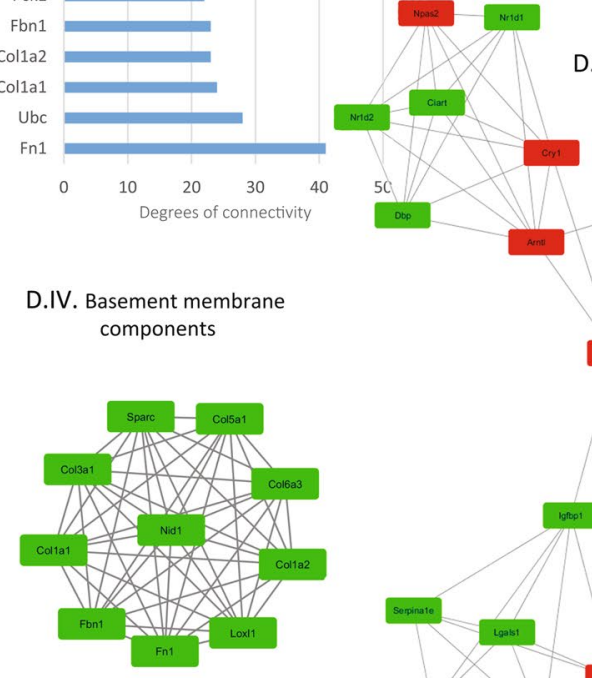

D.III. Circadian Rhythm

D.I. Regulation of lypolisis

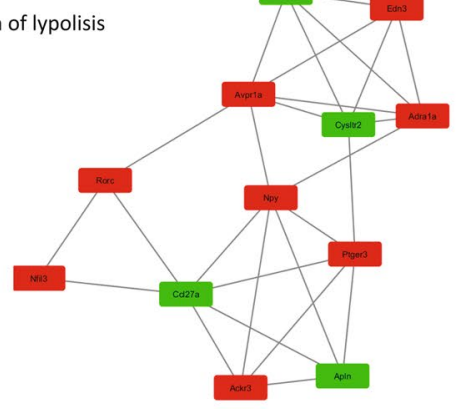

\section{D.tir. Circadian Rhyt}

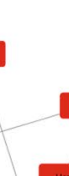
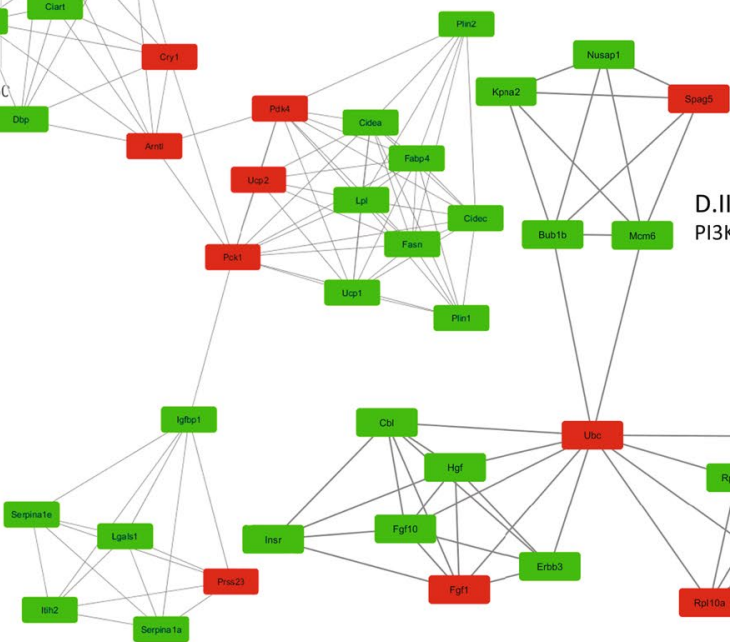
D.Il. Ribosome make-up.
PI3K-Akt signaling pathway

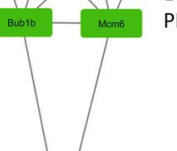

Figure 7. Kidney analysis. (A) Protein-protein interaction network generated with STRING from DEGs in the kidney. The network comprises 430 nodes and connected with 812 edges (p-value of 1.0 e-16). For the rest of the details see Fig. 3 and also high resolution panel A in Supplementary information 2. (B) The graph shows the results from the pathway enrichment analyses for the DEGs obtained from the Kidney. The bars depict the negative logarithm of the false discovery rate (FDR). (C) Genes with the highest degree of connectivity obtained from the PPI network with Cytoscape. D.I-IV) Top four modules obtained from the PPI network using Cytoscape's plugin MCODE (Red nodes: Upregulated in hepta KO mice; Green nodes: Downregulated in heptaKO mice).

The creation of an animal model devoid active TRPC genes, the TRPC heptaKO mouse, gave us an opportunity to address changes in gene expression in several tissues simultaneously, and to evaluate at a global level which functions depend on TRPC channels during their normal functioning. Based on the 4305 differentially 
A

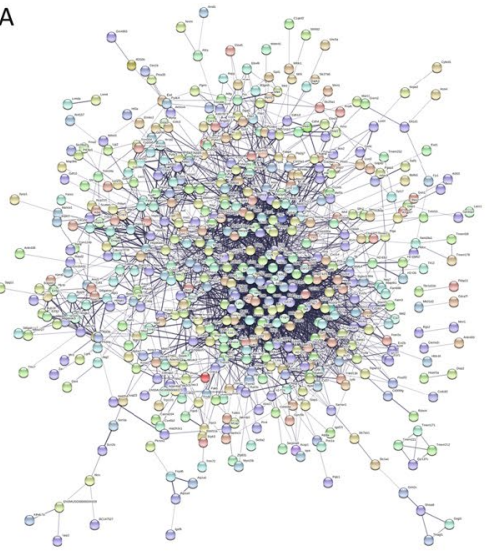

C

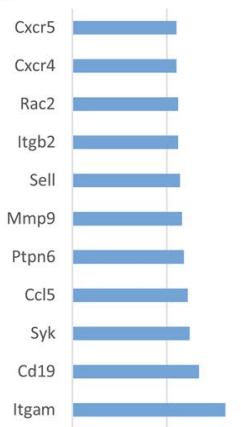

Itgam 50

D.I. Cytokine-cytokine
receptor interaction nteraction.
B

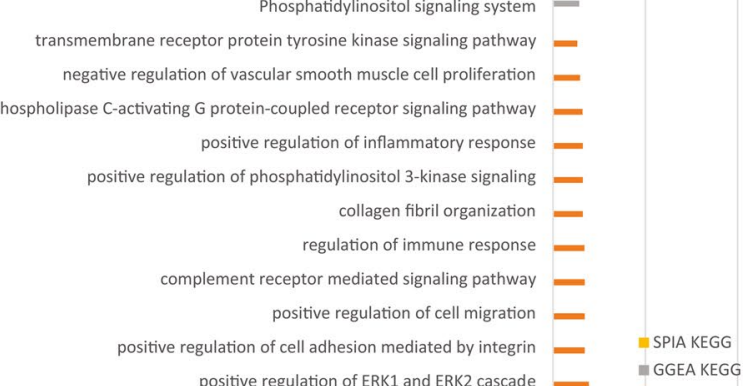

positive regulation of ERK1 and ERK2 cascade _ $\quad$ GGEA KEGG

leukocyte cell-cell adhesion ORA GO

cell chemotaxis

cell surface receptor signaling pathway

integrin-mediated signaling pathway

\section{(1)}

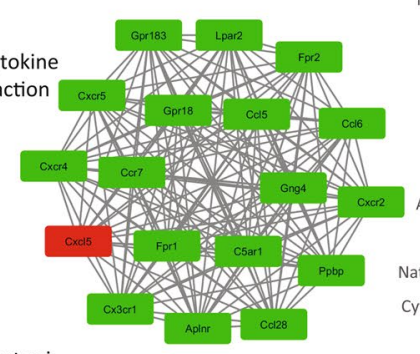

D.II. ECM-receptor i Basement membrane
composition. ane
Cell adhesion

PI3K-Akt signaling pathway

ECM-receptor interaction

Circadian Rythm

Protein digestion and absorption

Antigen processing and presentation

PPAR signaling pathway

atural killer cell mediated cytotoxicity

cytokine-cytokine receptor interaction

Cell adhesion molecules (CAMs)

Chemokine signaling pathway

Hematopoietic cell lineage

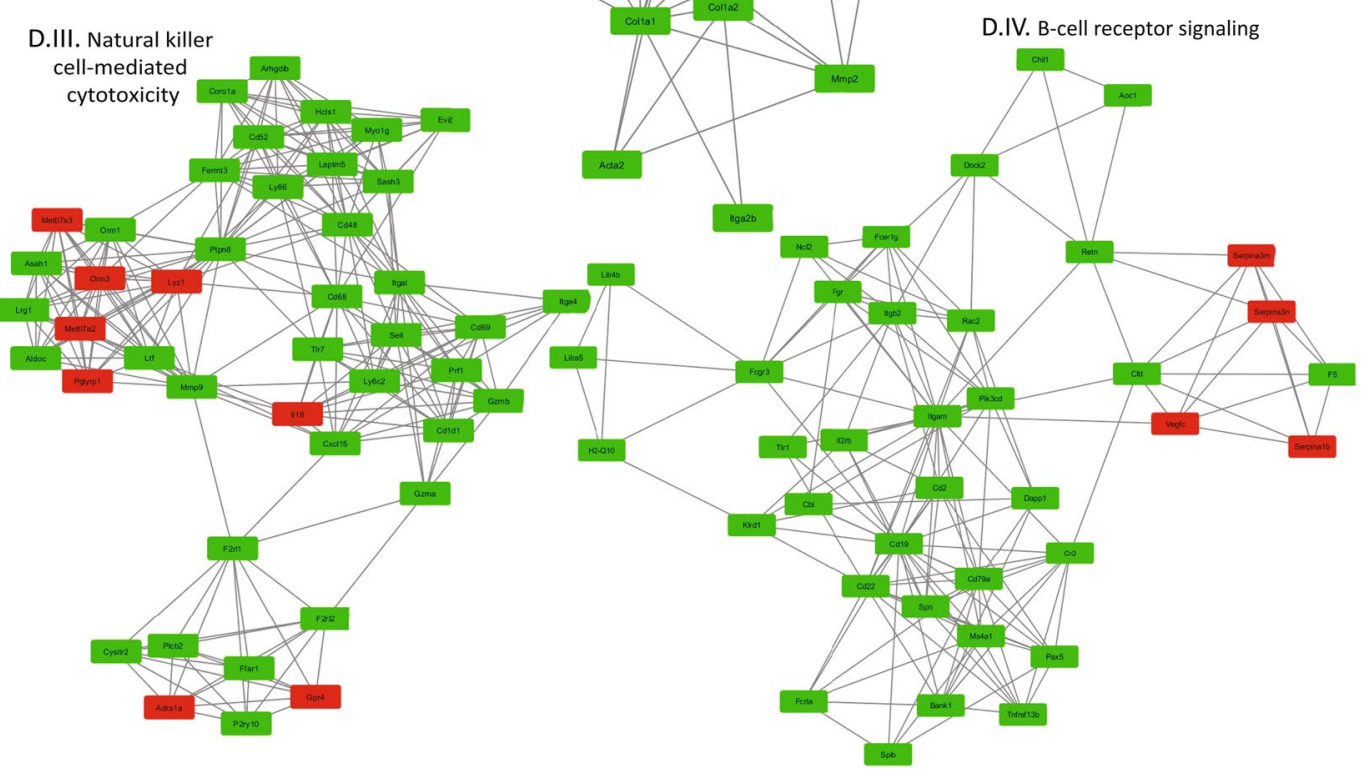

Figure 8. Lung analysis. (A) Protein-protein interaction network generated with STRING from DEGs in the lung. The network comprises 658 nodes and connected with 2945 edges (p-value of 1.0 e-16). For the rest of the details see Fig. 3 and also high resolution panel A in Supplementary information 2. (B) The graph shows the results from the pathway enrichment analyses for the DEGs obtained from the lung. The bars depict the negative logarithm of the false discovery rate (FDR). (C) Genes with the highest degree of connectivity obtained from the PPI network with Cytoscape. D.I-IV) Top four modules obtained from the PPI network using Cytoscape's plugin MCODE (Red nodes: Upregulated in hepta KO mice; Green nodes: Downregulated in heptaKO mice).

expressed genes in one or more of the tissues examined we performed an enrichment analysis using the KEGG and GO databases, which assemble combinations of expressed genes into diverse well defined cellular processes, and asked which processes were affected in more than one of the tissues of which we analyzed the transcriptome 


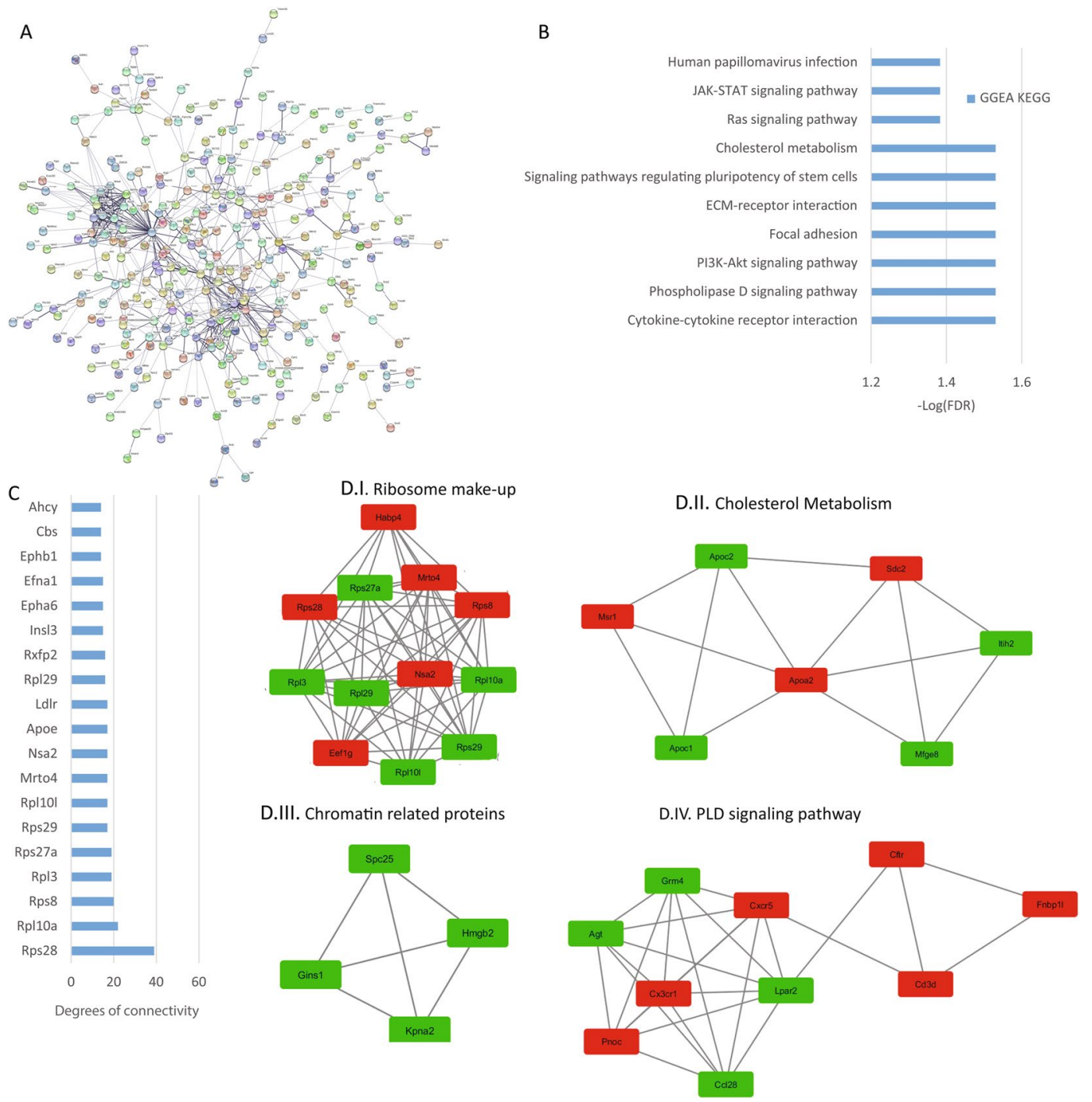

Figure 9. Testis analysis. (A) Protein-protein interaction network generated with STRING from DEGs in the testis. The network comprises 483 nodes and connected by 582 edges (p-value of 0.0003 ). For the rest of the details see Fig. 3 and also high resolution panel A in Supplementary information 2. (B) The graph shows the results from the pathway enrichment analyses for the protein-coding DEGs obtained from the testis. The bars depict the negative logarithm of the false discovery rate (FDR). (C) Genes with the highest degree of connectivity obtained from the PPI network with Cytoscape. D.I-IV) Top four modules obtained from the PPI network using Cytoscape's plugin MCODE (Red nodes: Upregulated in hepta KO mice; Green nodes: Downregulated in heptaKO mice).

by RNA-seq. We obtained a list of affected pathways that might explain several phenotypes observed in the different $\mathrm{KO}$ models of TRPC. The most relevant are discussed next.

One of the pathways we found to be modified is the ECM-receptor interaction. The extracellular matrix is a complex three-dimensional network with multiple components in which cells are located. It is mainly composed of macromolecules such as collagen, elastin, fibronectin, laminins, glycoproteins, proteoglycans and glycosaminoglycans, which are highly acidic and hydrated molecules many of which interact with cell surface receptors. The ECM acts as a physical scaffold interacting with cells through surface receptors, such as integrins, discoidin domain receptors, cell surface proteoglycans, and the hyaluronan receptor CD44. In this way, ECM regulates many cellular processes among them growth, migration, differentiation, survival, homeostasis, and morphogenesis are the most important ${ }^{48,49}$. Changes in the ECM composition are related to various syndromes and pathologies $^{50}$. In this regard, the microenvironment, in which diseases progress, is nowadays considered crucial for the development of pathologic conditions $s^{51,52}$. Some specific forms of interaction between ECM and cells are called focal adhesion or focal contacts. These structures, many transmembrane receptors, form the anchor of cellular actin filaments. Other components of focal adhesions are protein kinases and phosphatases that govern signaling pathways that eventually define the cellular processes. Though there is little bibliography supporting the presence of TRPC proteins in cell-ECM junctions, recent work suggests that TRPC7 colocalizes and interacts with syndecan-4 (part of focal adhesion complexes). TRPC7 has been shown to be located in focal adhesions with a 
A

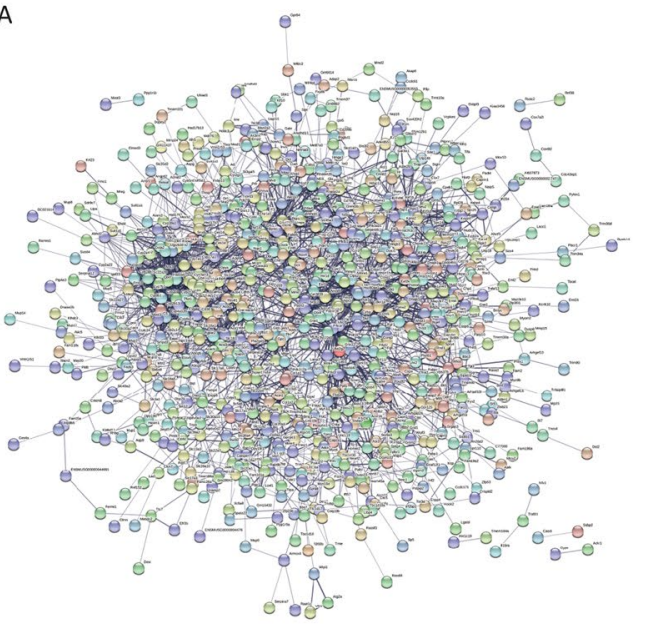

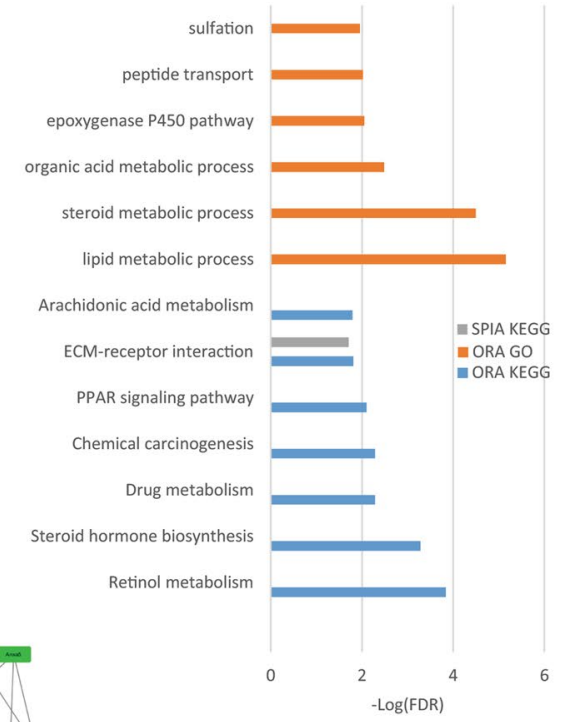

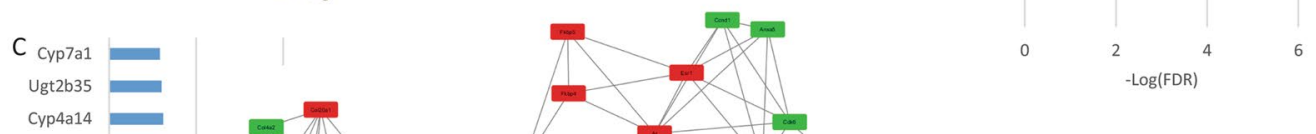

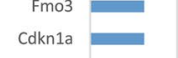

Cyp2c38

Esr1

Hspa1b

Cyp2b10

Cyp2b13

Hsd $3 \mathrm{bb5}$

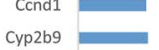

Hsp90aa1

Ubc $\begin{array}{lcl}0 & 50 \quad 100 \\ \text { Degrees of connectivity }\end{array}$
D.I. ECM- components. PI3K-Akt signaling
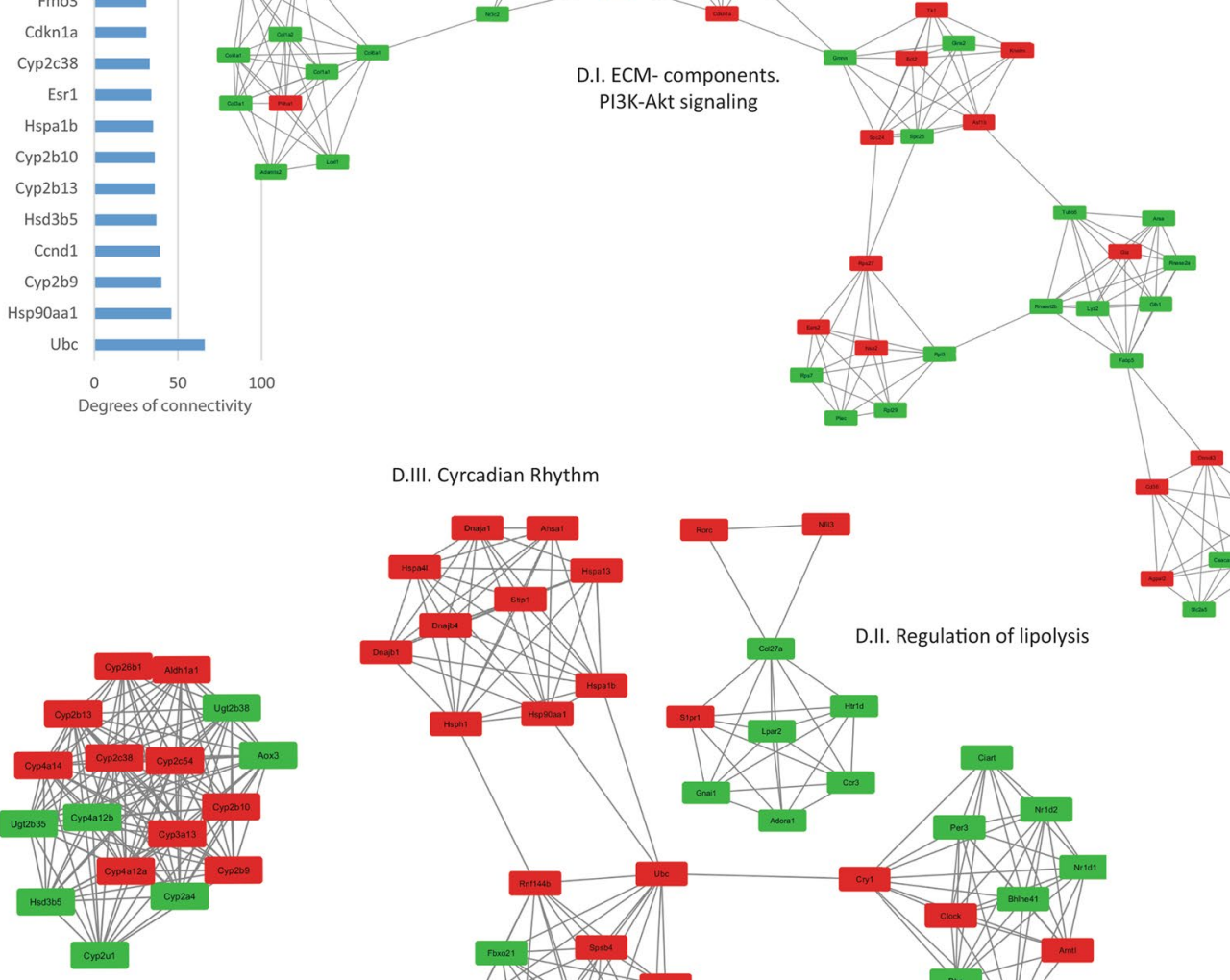

D.III. Cyrcadian Rhythm

D.IV. Retinol metabolism
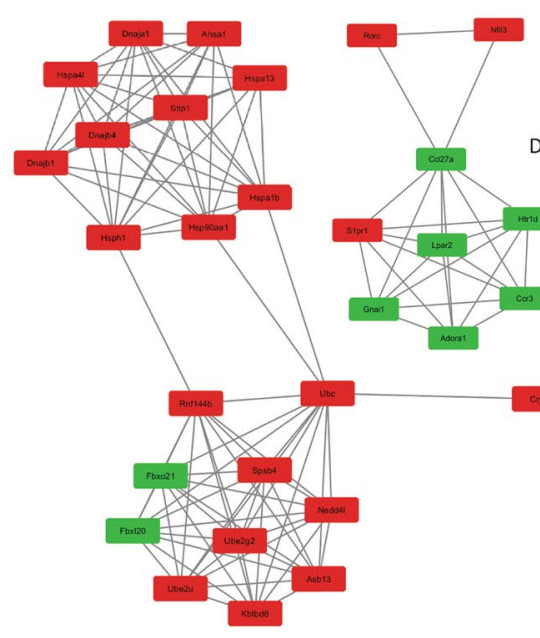

D.II. Regulation of lipolysis
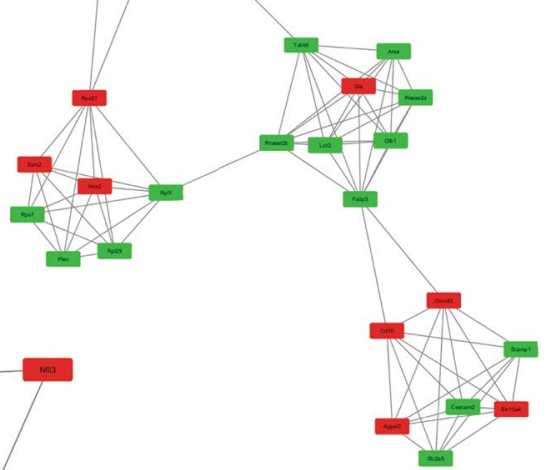

.

Figure 10. Liver analysis. (A) Protein-protein interaction network generated with STRING from DEGs in the Liver. The network comprises 937 nodes and connected with 2691 edges (p-value of 1.0 e-16). For the rest of the details see Fig. 3 and also high resolution panel A in Supplementary information 2. (B) The graph shows t the results from the pathway enrichment analyses for the DEGs obtained from the liver. The bars depict the negative logarithm of the false discovery rate (FDR). (C) Genes with the highest degree of connectivity obtained from the PPI network with Cytoscape. D.I-IV) Top four modules obtained from the PPI network using Cytoscape's plugin MCODE (Red nodes: Upregulated in hepta KO mice; Green nodes: Downregulated in heptaKO mice).

similar pattern to that of alpha-actinin-1. Syndecan-4 KO mice show a reduced number of stress fibers and focal adhesions, an effect that can be reversed by removing TRPC7, thus, suggesting that the abnormalities observed were a direct effect of the interaction of Syndecan 4 and TRPC7. Also, consistent with roles in cell adhesion 
A

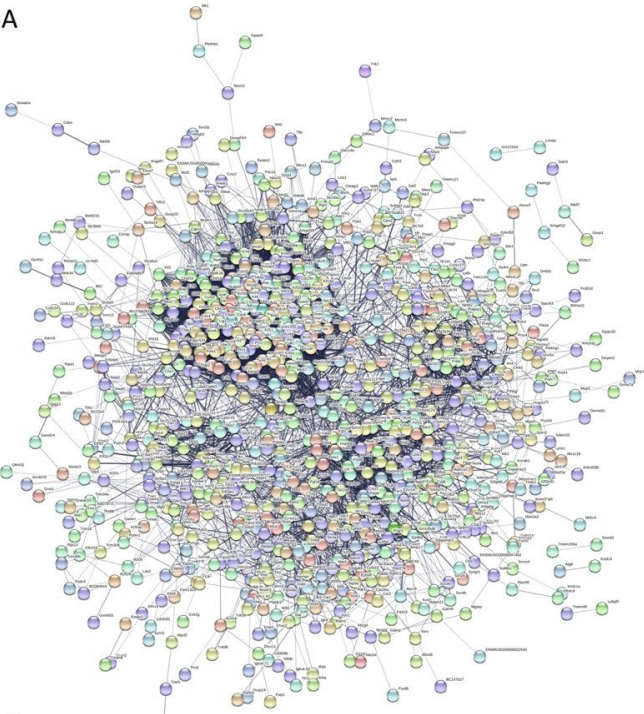

C

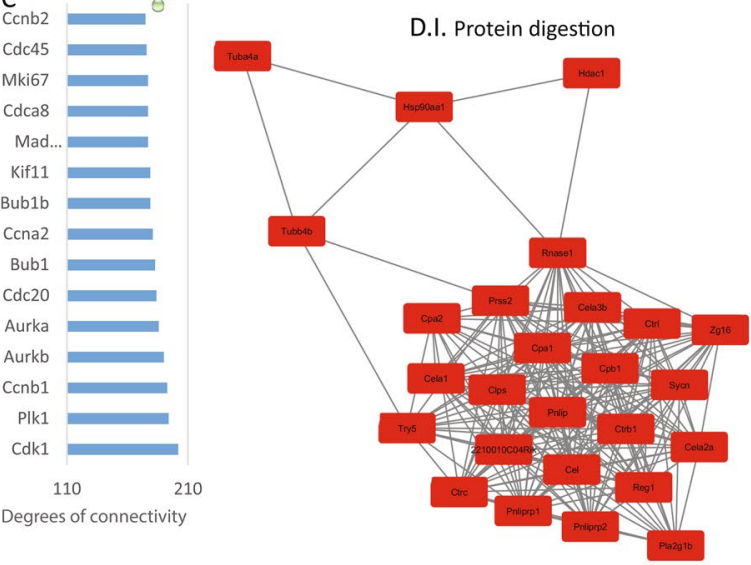

D.III. Cytokine Related proteins

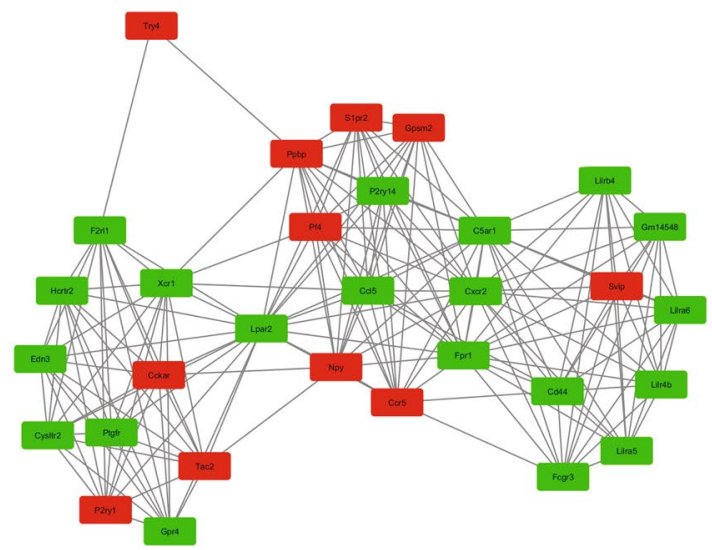

B

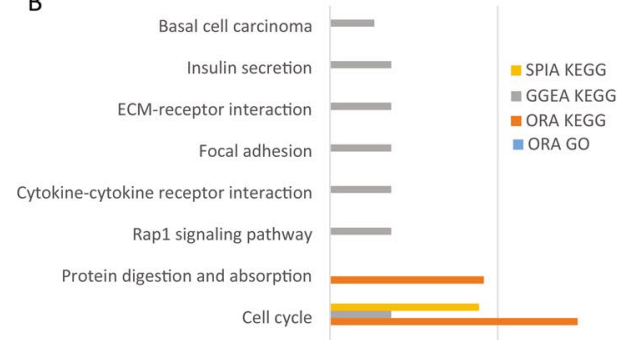

positive regulation of MAP kinase activity

positive regulation of cell cycle

mitotic spindle assembly checkpoint

Cell adhesion

erythrocyte development

positive regulation of ERK1 and ERK2 cascade

DNA replication

Cell division

0

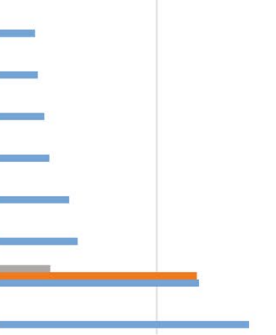
5
$-\log (F D R)$

D.II. Ribosome make-up

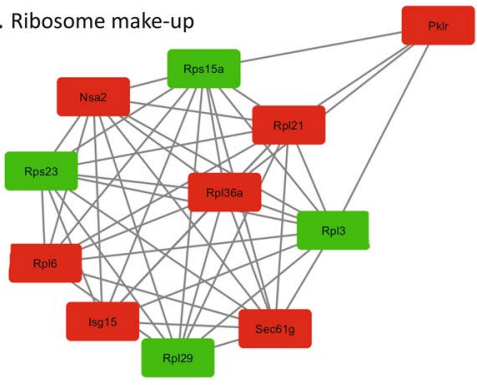

D.IV. Cell cycle and DNA replication

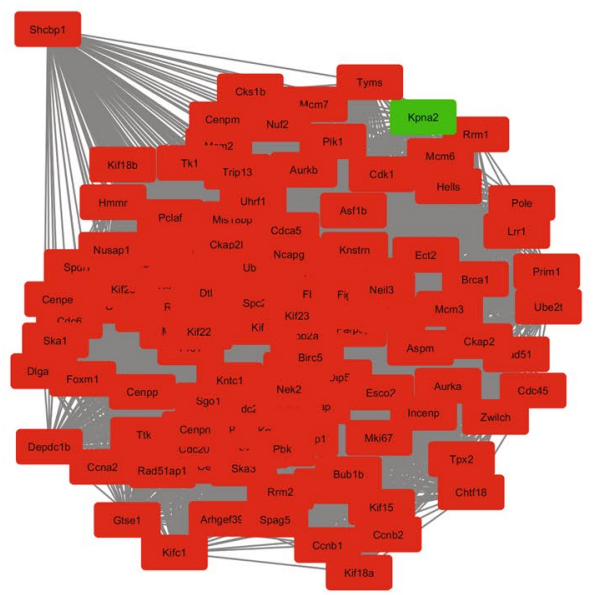

Figure 11. Spleen analysis. (A) Protein-protein interaction network generated with STRING from DEGs in the spleen. The network comprises 1015 nodes and connected with 14204 edges ( $p$-value of 1.0 e-16). For the rest of the details see Fig. 3 and also high resolution panel A in Supplementary information 2. (B) The graph shows the results from the pathway enrichment analyses for the DEGs obtained from the spleen. The bars depict the negative logarithm of the false discovery rate (FDR). (C) Genes with the highest degree of connectivity obtained from the PPI network with Cytoscape. D.I-IV) Top four modules obtained from the PPI network using Cytoscape's plugin MCODE (Red nodes: Upregulated in hepta KO mice; Green nodes: Downregulated in heptaKO mice).

and junction formation, depletion of TRPC4 increased the expression of P-cadherin (cadherin 3) in adherens junctions $s^{53}$. It is important to mention that Darier's disease and psoriatic epidermis that show altered calcium homeostasis also show altered expression of both, P-cadherin and TRPC ${ }^{54-57}$. Another study found that TRPC5 
A

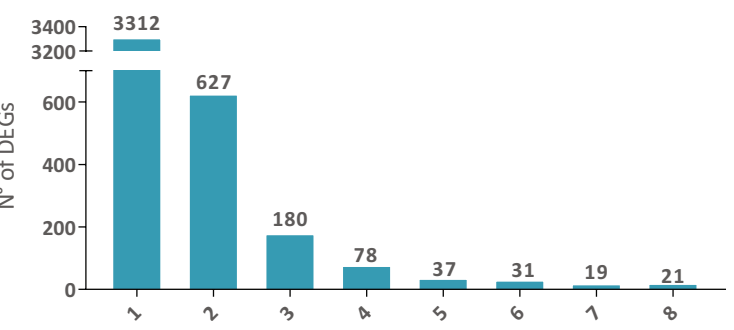

B
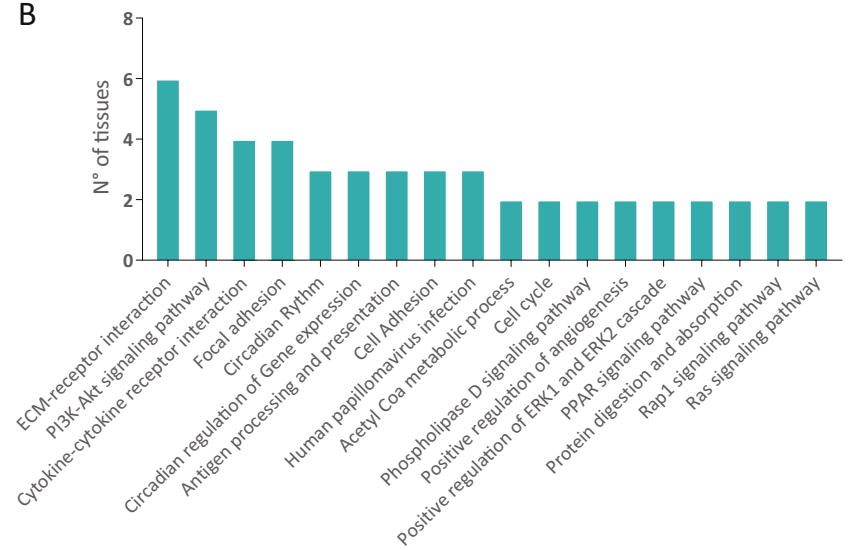

Figure 12. (A) Number of differentially expressed genes. Bars depict the number of DEGs found in the indicated number of tissues. (B) Enriched pathways most represented in all the tissues analyzed. The bars depict the number of tissues in which the indicated pathways are significantly modified.

\begin{tabular}{|l|l|}
\hline Gene Symbol & Description \\
\hline Gdpd3 & glycerophosphodiester phosphodiesterase domain containing 3 \\
\hline Trim12a & tripartite motif-containing 12A \\
\hline mt-Tg & mitochondrially encoded tRNA glycine \\
\hline Padi4 & peptidyl arginine deiminase, type IV \\
\hline Rpl29 & ribosomal protein L29 \\
\hline Trim30d & tripartite motif-containing 30D \\
\hline Eif3j1 & eukaryotic translation initiation factor 3, subunit J1 \\
\hline mt-Nd3 & mitochondrially encoded NADH dehydrogenase 3 \\
\hline Kpna2 & karyopherin (importin) alpha 2 \\
\hline Imjd7 & jumonji domain containing 7 \\
\hline Capza1 & capping protein (actin filament) muscle Z-line, alpha 1 \\
\hline Trim34a & tripartite motif-containing 34A \\
\hline Zfp457 & zinc finger protein 457 \\
\hline Ahcy & S-adenosylhomocysteine hydrolase \\
\hline Naip5 & NLR family, apoptosis inhibitory protein 5 \\
\hline
\end{tabular}

Table 2. DEGs Shared by all Tissues.

channels interact through alpha5beta1 integrin with GM1 ganglioside. The integrin then induces the autophosphorylation of its associated focal adhesion kinase (FAK), which in turn activates PLC and PI3K ${ }^{58}$. Some TRPC channels can be activated by the stretching of the membrane ${ }^{59,60}$. TRPC 5 and TRPC 6 sensing could thus work as osmotic sensor signals to restore hydromineral balance ${ }^{7,61,62}$. TRPC1, for example, is localized in caveolae that are associated with cell shape and actin-controlled changes in membrane tension ${ }^{63}$. The ability to sense changes in the tension of the membrane might thus regulate the formation of focal adhesions.

ECM receptor interactions and focal adhesions are both related to various cellular processes, one of the most important being migration. Migration requires turnover of cell-ECM adhesion complexes with continuous cytoskeleton rearrangement and alterations of cell shape. TRPCs could act in regulating calcium homeostasis and interacting with cell adhesion molecules and other signaling proteins ${ }^{64}$. In this regard, the role of TRPC1 in migration has been described by several groups ${ }^{65-67}$. Upon knockdown of TRPC1, EGF-mediated chemotactic migration is inhibited ${ }^{68}$. TRPC6, on the other hand, promotes cancer cell migration in head and neck squamous cell carcinomas and in glioblastoma ${ }^{69,70}$. 
The results obtained here highlight the importance of TRPCs in many functions that depend on the interaction of the ECM with the cells. This allows us to hypothesize a role of TRPCs in cancer progression and other pathologies.

Several studies relate TRPCs to PI3K-Akt signaling pathways. The PI3K/Akt signaling pathway is a major intracellular pathway that among other cellular processes regulates survival, cell growth, differentiation, cellular metabolism, and cytoskeletal reorganization of cells in response to different signals. Akt is downstream of PI3K and is activated through phosphorylation of two residues (serine 473 and threonine 308). PI3Ks are activated by growth factors through GPCR or RTK receptors. The PI3K activation results in the conversion of PtdIns(4,5)P2 (PIP2) to PtdIns(3,4,5)P3 (PIP3), a process that is reversed by the phosphatase PTEN. Then PIP3 interacts with the $\mathrm{PH}$ domain of Akt causing its retention at the plasma membrane where it is phosphorylated by 3-phosphoinositide-dependent protein kinase 1 (PDK1) at Thr308 and by PDK2 at Ser 473 resulting in activation of Akt's kinase activity. Akt subsequently phosphorylates a variety of proteins that lead to different cellular responses ${ }^{71,72}$. This pathway is also dysregulated in various pathologies such as different types of cancer and augmented drug resistance ${ }^{73}$. Activation of TRPC6 in bronchial epithelial cells by LPS/TLR4 and in renal glomerular mesangial cells through cAMP involves PI3K/Akt ${ }^{74,75}$. Evidence also shows that PI3K-mediated signaling pathways are essential in endothelin A (ETA) receptor-evoked TRPC1/C5/C6 and TRPC3/C7 channel activation $^{76}$. In agreement with this, soluble Klotho (klotho) protein present in the systemic circulation inhibits TRPC6 currents in cardiomyocytes by blocking phosphoinositide-3-kinase-dependent exocytosis of TRPC6 channels ${ }^{77}$. Moreover, TRPC1, TRPC5, and TRPC6 translocation to the plasma membrane was found to be dependent on PI3K pathways in different types of cell $s^{61,78-80}$. These studies suggest an upstream role for TRPCs activating the PI3K/Akt pathway. In agreement, activating TRPC6 with hyperforin leads to the activation of Ras/MEK/ERK, PI3K/Akt, and CAMKIV kinases and these cues are necessary to promote TRPC6 mediated synaptic plasticity in PC12 cells and primary hippocampal neurons ${ }^{81}$. Also, VEGF can activate TRPC3 and this is followed by PI3K/ Akt activation ${ }^{82}$. The administration to mice of M084, an inhibitor of TRPC4/5 increased the phosphorylation levels of Akt and ERK in the prefrontal cortex ${ }^{83}$. Consistent with these findings from our laboratory showed that in cardiomyocyte cultures from triple $\mathrm{KO}$ mice (TRPC3/6/7 -/-) pAkt was reduced compared to $\mathrm{WT}^{16}$. Also, TRPC1 was found to regulate positively pAkt in PTEN deficient breast cancer cell lines and the lack of TRPC1 or the inhibition of calcium entry reduces Akt phosphorylation and delays muscle cell differentiation ${ }^{84,85}$. Though the evidence does not allow for resolution as to whether TRPC6 activates PI3K/Akt or it is the other way around, it is clear that there is a direct link between the PI3K/Akt pathway and the TRPC protein family.

To our knowledge, there is no experimental data that relate to TRPC channels with circadian rhythm in mammals to date. For this reason, the results presented here are novel and present a new possible function for these proteins not previously reported. The circadian rhythms are periodic oscillations that occur within 24 hours and determine the sleeping and awakening times, affecting, thus, the whole organism. They are commonly known as an internal biological clock that has components that are common among species and others that vary ${ }^{86}$. These internal clocks work in mammals as negative and positive transcriptional-translational feedback loops where the first negative feedback loop is a rhythmic transcription of period genes (PER1, PER2, and PER3) and cryptochrome genes (CRY1 and CRY2). PER and CRY proteins form a heterodimer, which acts on the CLOCK/ BMAL1 heterodimer to repress its own transcription ${ }^{87}$. Phosphorylation of PER and CRY proteins by casein kinase epsilon (CKIepsilon), produces their degradation and restarts the cycle. Then a positive feedback loop initiates the transcription of genes that contain E-box cis-regulatory enhancer sequences and involves CLOCK/ BMAL1 heterodimer ${ }^{87,88}$. Reinforcing our findings, it is worth noting that a function for TRPC homologs in Drosophila circadian rhythm has been previously reported ${ }^{89}$. On the other hand, the oscillator circuits do not include a precise timer and constant darkness or constant light leads animals to lose their circadian rhythms. Entrainment and maintenance of circadian rhythms depend on two TRPCs, TRPC6, and TRPC7 which are activated in intrinsically photosensitive Retinal Ganglion Cells (ipRGCs) that project to the suprachiasmatic nucleus (SCN). In ipRGCs light-sensitive melanopsin (Opsin4), a Gq protein-coupled receptor activates phospholipase Cbeta4, which in turn leads to activation of the heteromeric TRPC6/7 channel and cell depolarization ${ }^{20}$. The resulting action potential drives the activity of neurons of the SCN in which the levels of the PER, CRY, CLOCK, and BMAL1 proteins oscillate. In mammals, circadian rhythm controls several processes including heartbeat, sleep-wake cycles, and functions of the immune system and metabolism ${ }^{90}$. Our results show important alterations in the expression of the clock genes but if these changes would result in an altered circadian rhythm needs be studied in the animal model for further corroboration and to determine and describe the phenotype(s) associated with such a dysregulation.

Modifications in intracellular calcium concentrations represent a fundamental mechanism in the control of inflammatory and immune cell functions and the study of the role of TRPC in the innate and adaptive immune response has grown in the last years. One of the pathways modified in the TRPC KO mice is Cytokine-Cytokine receptor interaction. Recent evidence has shown a role for TRPC in several cellular mechanisms of potential significance for the pathophysiology of the innate immune response. TRPC1 in the endothelium increases vascular permeability after TNF/thrombin stimulation ${ }^{91}$. Studies in animal models show that TRPC1 may control IL1 $\beta$ release from macrophages ${ }^{92}$. Similarly, TRPC1 appears to affect the late effects of anaphylaxis by controlling TNF release from mast cells ${ }^{93}$. Another study suggests that mechanical stretch may induce an influx of Calcium and up-regulation of IL-13 and MMP-9 expression in 16HBE cells via activation of TRPC1 ${ }^{94}$. TRPC1 is also involved in the inflammatory response to bacterial infection through the TLR4/TRPC1/NF-kB signaling route ${ }^{95}$. In addition, TRPC1 appears to contribute to the regulation of the epithelial-mesenchymal transition (EMT) in cancer; its inhibition suppresses TGF- $\beta 1$-induced $\mathrm{EMT}^{96,97}$. TRPC3 is expressed in various immune cells and has been proposed to contribute to vascular inflammation ${ }^{98-100}$. Also, the brain-derived neurotrophic factor (BNDF) has the effect of upregulating TRPC3 in the cell membrane. This effect has been proposed to avoid neuronal inflammation and myocardial injury ${ }^{101-103}$. Monocytes from patients with hypertension showed an increased TRPC3 
expression that was significantly associated with increased expression IL-1beta or TNF-alpha ${ }^{104}$. Alawi et al. found that TRPC5 in inflammatory joint conditions was related to an endogenous anti-inflammatory/analgesic pathway. The same study determined that in human inflammatory arthritis chronic treatment with a TRPC5 antagonist reduced TRPC5 mRNA at the same time that augmented the asymmetry, and elevated cytokine concentrations ${ }^{105}$. TRPC 5 channels are do not express in resting CD4+ and CD8+ T-cells but are hyper expressed activated cells where they increase Calcium influx ${ }^{106}$. TRPC6 is the most widely studied TRPC in relation to the immune system. TRPC6 is expressed in various cell types (neutrophils, lymphocytes, platelets, and endothelium $)^{107}$. TRPC6 has a role in neutrophil mobilization and promotes the loss of endothelial junctions during acute inflammation ${ }^{108-111}$. In animal models histamine-induced vascular leakage is dependent on TRPC6 ${ }^{112}$. TRPC6 cooperates in lipopolysaccharide-induced endothelial activation after being itself activated by increasing intracellular concentrations of diacylglycerol (DAG) downstream of the activation of Toll-like receptor 4 (TLR $4^{113}$ ). Using a human intestinal myofibroblast model and biopsy samples from patients with cardiovascular disease (CD)), it was shown that although increased TRPC6 (and presumably TRPC4) activity promotes the TGFbeta1-mediated expression of alpha-Smooth Muscle Actin (a-SMA) and N-cadherin and strengthens interactions between the 2 molecules, it also negatively regulates collagen synthesis and the secretion of antifibrotic factors, such as IL-10 and IL-11. TGF-beta1-increased Calcium influx through TRPC6 channels, regulating negatively TGF-beta1-Smad/ p38-MAPK/ERK1/2 signaling through calcineurin activation ${ }^{114}$. It was also described that TRPC6 channels in Human Hepatocellular Carcinoma Cells are involved in migration and invasion in response to TGF- $\beta^{115}$. Cytokine complexes are widely studied as target molecules for different pathologies. The difficulty in their use will be that the many functions cytokines have can lead to undesirable secondary effects.

This is why targeting TRPC proteins might be of interest to modulate immune responses and inflammations driven by cytokine-cytokine receptor interaction.

\section{Conclusion}

There is a vast bibliography relating TRPC channels to different pathologies many of which were obtained from in vivo analysis of KO mice. For example, TRPC1 KO mice showed reduced secretion of salivary fluid ${ }^{116}$, a modest decrease in osteoclastogenesis and an increase in bone mass ${ }^{117}$, and movement deficits related to widespread neuronal loss including dopaminergic neurons in basal ganglia ${ }^{118}$. TRPC2 is a pseudogene in humans but in mice is highly expressed in the sensory terminals of the vomeronasal organ. Coherently, the lack of TRPC2 leads to behavioral changes such as loss of sexual discrimination, and impaired maternal aggression (offspring protection) ${ }^{119,120}$. TRPC3 knockout mice show impaired motor coordination ${ }^{121}$ and TRPC6 knockout mice display reduced litter sizes and structural changes of the placenta ${ }^{122}$. All these results led us to hypothesize that mice lacking all seven TRPCs would probably die at early embryonic age or at least have severe developmental defects. To our surprise TRPC heptaKO mice are alive and breeding, through extensive analyses have not been done as yet. Nonetheless, through the present RNA-seq analysis we detected several pathways that are dysregulated compared to the WT mice which however do not lead to the death of the mice. Having said this, we need to keep in mind that many pathologies are related to failure in the transport of calcium through the PM and the ER and many involve increases in intracellular calcium.

We found several pathways dysregulated both in general and in a tissue-specific way. Our work provides the first description of TRPC KO mice at the RNA level providing a starting point to understand the function of TRPC channels and their possible roles in pathologies where their expression is affected.

\section{Materials and Methods}

Experimental animals. Wild type mouse strains were either 129SvEv, C57Bl/6J or crossbreeds between 129SvEv and C57Bl/6J. TRPC2-/ - mice and TRPC4-/ - mice were backcrossed for more than 5 generations to $\mathrm{C} 57 \mathrm{Bl} 6 \mathrm{~J}$ or $\mathrm{C} 57 \mathrm{Bl} / 6 \mathrm{~N}$, respectively and then crossed into $129 \mathrm{SvEv} \mathrm{xC} 57 \mathrm{Bl} / 6 \mathrm{~J}$ mice carrying the disrupted alleles of combinations of the TRPC1, TRPC3, TRPC5, TRPC6, and TRPC7 genes. The creation of the individual disrupted TRPC alleles and their validation has been previously described (TRPC1 ${ }^{32}$; TRPC2 ${ }^{120}$; TRPC $3^{121}$; TRPC4 ${ }^{123}$; TRPC ${ }^{124}$; TRPC6 ${ }^{125}$; TRPC $^{126}$ ).

Mice were housed in the Association for Assessment and Accreditation of Laboratory Animal Care (AAALAC)-accredited, specific pathogen-free vivarium of the Comparative Medicine Branch of the NIEHS. Animals were treated in compliance with the Guide for the Care and Use of Laboratory Animals (National Academy of Sciences). All procedures and crosses to which the mice were subjected were approved by the NIEHS's Animal Care and Use Committee (ACUC).

Isolation of RNA from tissues. Four TRPC KO and four WT three-month-old male mice were used to obtain RNA samples from 7 tissues: Heart, Kidney, Spleen, Testis, Liver, Lung and Brain. The brain was split into two fractions: Forebrain and the remainder of the CNS including brainstem and cerebellum collectively referred to as MidBrain for a total of 64 samples. In several cases, the Forebrain and Midbrain data were pooled and are presented as Brain Mice were euthanized in $\mathrm{CO} 2$ chambers and their tissues rapidly dissected, rinsed in ice-cold Dulbecco's PBS, placed into ice-cold 15-mL Corex tubes with aliquoted RLT solution supplied in RNAeasy Kits (QIAGEN cat \#74106) and homogenized with the aid of a Polytron homogenizer fitted with a PT-10 generator. Total RNA was isolated following RNAeasy Kit instructions which include on-column DNAse treatment and sent to Expression Analysis (Research Triangle Park, North Carolina (www.ExpressionAnalysis.com)) for sequencing by the paired-end method. RNA quality was assessed by electrophoresis and bioanalyzer prior to RNA sequencing. An input of $100 \mathrm{ng}$ of total RNA was used to construct cDNA libraries (TruSeq Stranded mRNA Sample Prep Kit, Illumina, \#RS-122-2103) following the manufacturer's instructions. The data analyzed during the current study was deposited in the National Centre for BiotechnologyInformation-Sequence Read Archive (NCBI-SRA) repository with the accesion number PRJNA606559 (www.ncbi.nlm.nih.gov/sra/PRJNA606559). 
Twenty-five million 51 bp reads were obtained by the RNA-seq paired-end method with Illumina Bioanalyzer technology. After sequencing, raw data were obtained in the fastq format. FastQC was used for validating the quality of the data. To correct read errors/bias Samtools and trimgalore were used. The data was then aligned to genome ensembl38 using STAR. In order to visualize the variation in expression between samples, we performed a Principal Component Analysis (PCA) using the programming environment R.

Differential gene expression. The quantification of number and density of reads at the gene level, as well as the differential expression analysis, were carried out under contract by Ariel Chernomoretz, Fundación Instituto Leloir, Buenos Aires,Argentina, with the software ASpli (https://bioconductor.org/packages/release/ bioc/html/ASpli.html) and the edgeR package of the statistical programming environment R. The DEGs were identified requiring a fold change greater than $50 \%$ and an adjusted $\mathrm{p}$-value (Hochberg-Benjamini multiple test correction) to achieve a false discovery rate $<0.01$ (adjusted $\mathrm{p}$-value $<0.01$ ) $^{127}$.

Protein-protein interaction and pathway enrichment analysis. The protein-protein interaction (PPI) networks were constructed using STRING (https://string-db.org/) for each tissue ${ }^{19}$. Next, we used the Cytoscape software ${ }^{128}$ to obtain the top hub proteins were selected from the network. The molecular complex detection plug-in (MCODE) was used to obtain the modules. The criteria used were Degree cut-off $=2$, node score cut-off $=0.2, \mathrm{k}$-core $=2$, and $\max$ Depth $=100$. The top modules were selected for graphical representation.

The pathway enrichment analysis was performed with two strategies implemented in the EnrichmentBrowser and cluster profile packages: the over-representation analysis (ORA) and the network-based gene set enrichment analysis (NGSEA). The ORA analysis was performed both for GO and KEGG. On the other hand, the NGSEA analysis was performed with the Gene Graph Enrichment Analysis (GGEA) and the Signaling Pathway Impact Analysis (SPIA) using KEGG pathways for both cases. The pathways considered significantly changed had a false discovery rate $(\mathrm{FDR})<0.05$.

Received: 18 December 2019; Accepted: 21 February 2020;

Published: 29 April 2020

\section{References}

1. Zhu, X. et al. trp, a novel mammalian gene family essential for agonist-activated capacitative Ca2+entry. Cell 85, 661-671 (1996).

2. Putney, J. W. The enigmatic TRPCs: multifunctional cation channels. Trends Cell Biol. 14, 282-286 (2004).

3. Vazquez, G., Wedel, B. J., Aziz, O., Trebak, M. \& Putney, J. W. The mammalian TRPC cation channels. Biochim. Biophys. Acta - Mol. Cell Res. 1742, 21-36 (2004).

4. Vangeel, L. \& Voets, T. Transient receptor potential channels and calcium signaling. Cold Spring Harb. Perspect. Biol. 11 (2019).

5. Hilton, J. K., Kim, M. \& Van Horn, W. D. Structural and Evolutionary Insights Point to Allosteric Regulation of TRP Ion Channels. Acc. Chem. Res. 52, 1643-1652 (2019).

6. Madej, M. G. \& Ziegler, C. M. Dawning of a new era in TRP channel structural biology by cryo-electron microscopy. Pflugers Archiv European Journal of Physiology 470, 213-225 (2018).

7. Mederos y Schnitzler, M. et al. Gq-coupled receptors as mechanosensors mediating myogenic vasoconstriction. EMBO J. 27, 3092-103 (2008).

8. Storch, U. et al. Dynamic NHERF interaction with TRPC4/5 proteins is required for channel gating by diacylglycerol. Proc. Natl. Acad. Sci. USA 114, E37-E46 (2017).

9. Svobodova, B. \& Groschner, K. Mechanisms of lipid regulation and lipid gating in TRPC channels. Cell Calcium 59, 271-279 (2016).

10. Saleh, S. N., Albert, A. P., Peppiatt-Wildman, C. M. \& Large, W. A. Diverse properties of store-operated TRPC channels activated by protein kinase C in vascular myocytes. J. Physiol. 586, 2463-2476 (2008).

11. Bröker-Lai, J. et al. Heteromeric channels formed by TRPC 1, TRPC 4 and TRPC 5 define hippocampal synaptic transmission and working memory. EMBO J. 36, 2770-2789 (2017).

12. Dhennin-Duthille, I. et al. High Expression of Transient Receptor Potential Channels in Human Breast Cancer Epithelial Cells and Tissues: Correlation with Pathological Parameters. Orig. Pap. Cell Physiol Biochem 28, 813-822 (2011).

13. Eder, P. \& Molkentin, J. D. TRPC Channels As Effectors of Cardiac Hypertrophy. Circ. Res. 108, 265-272 (2011).

14. Golovina, V. A. et al. Upregulated TRP and enhanced capacitative $\mathrm{Ca}(2+)$ entry in human pulmonary artery myocytes during proliferation. Am. J. Physiol. Heart Circ. Physiol. 280, H746-55 (2001).

15. Zeng, C., Tian, F. \& Xiao, B. TRPC Channels: Prominent Candidates of Underlying Mechanism in Neuropsychiatric Diseases. Mol. Neurobiol. 53, 631-647 (2016).

16. He, X. et al. Major contribution of the 3/6/7 class of TRPC channels to myocardial ischemia/reperfusion and cellular hypoxia/ reoxygenation injuries. Proc. Natl. Acad. Sci. 114, E4582-E4591 (2017).

17. Birnbaumer, L. From GTP and G proteins to TRPC channels: a personal account. J. Mol. Med. 93, 941-953 (2015)

18. Egorov, A. V. et al. TRPC channels are not required for graded persistent activity in entorhinal cortex neurons. Hippocampus 1-11. https://doi.org/10.1002/hipo.23094 (2019).

19. Szklarczyk, D. et al. STRING v11: Protein-protein association networks with increased coverage, supporting functional discovery in genome-wide experimental datasets. Nucleic Acids Res. 47, D607-D613 (2019).

20. Xue, T. et al. Melanopsin signalling in mammalian iris and retina. Nature 479, 67-72 (2011).

21. Sawamura, S., Shirakawa, H., Nakagawa, T., Mori, Y. \& Kaneko, S. TRP Channels in the Brain. Neurobiol. TRP Channels. https:// doi.org/10.4324/9781315152837-16 (2017).

22. Abramowitz, J. \& Birnbaumer, L. Physiology and pathophysiology of canonical transient receptor potential channels. FASEB J. 23, 297-328 (2009).

23. Niehof, M. \& Borlak, J. HNF4 alpha and the Ca-channel TRPC1 are novel disease candidate genes in diabetic nephropathy. Diabetes 57, 1069-77 (2008).

24. Goel, M., Zuo, C.-D. \& Schilling, W. P. Role of cAMP/PKA signaling cascade in vasopressin-induced trafficking of TRPC3 channels in principal cells of the collecting duct. Am. J. Physiol. Renal Physiol. 298, F988-96 (2010).

25. Sours-Brothers, S., Ding, M., Graham, S. \& Ma, R. Interaction between TRPC1/TRPC4 assembly and STIM1 contributes to storeoperated Ca2+ entry in mesangial cells. Exp. Biol. Med. (Maywood). 234, 673-82 (2009).

26. Schaldecker, T. et al. Inhibition of the TRPC5 ion channel protects the kidney filter. J. Clin. Invest. 123, 5298-5309 (2013). 
27. Sonneveld, R. et al. Glucose specifically regulates TRPC6 expression in the podocyte in an AngII-dependent manner. Am. J. Pathol. 184, 1715-26 (2014)

28. Goel, M., Sinkins, W. G., Zuo, C.-D., Estacion, M. \& Schilling, W. P. Identification and localization of TRPC channels in the rat kidney. Am. J. Physiol. Renal Physiol. 290, F1241-52 (2006).

29. Corteling, R. L. et al. Expression of transient receptor potential C6 and related transient receptor potential family members in human airway smooth muscle and lung tissue. Am. J. Respir. Cell Mol. Biol. 30, 145-54 (2004).

30. Ong, H. L., Brereton, H. M., Harland, M. L. \& Barritt, G. J. Evidence for the expression of transient receptor potential proteins in guinea pig airway smooth muscle cells. Respirology 8, 23-32 (2003).

31. Sweeney, M. et al. Role of capacitative Ca2+ entry in bronchial contraction and remodeling. J. Appl. Physiol. 92, 1594-602 (2002).

32. Dietrich, A. et al. Pressure-induced and store-operated cation influx in vascular smooth muscle cells is independent of TRPC1. Pflugers Arch. 455, 465-77 (2007).

33. Lu, W., Wang, J., Shimoda, L. A. \& Sylvester, J. T. Differences in STIM1 and TRPC expression in proximal and distal pulmonary arterial smooth muscle are associated with differences in Ca2+ responses to hypoxia. Am. J. Physiol. Lung Cell. Mol. Physiol. 295, L104-13 (2008).

34. Kumar, A. et al. Role of transient receptor potential channels in regulating spermatozoa functions: A mini-review. Vet. World 11, $1618-1623$ (2018)

35. Stamboulian, S. et al. Junctate, an inositol 1,4,5-triphosphate receptor associated protein, is present in rodent sperm and binds TRPC2 and TRPC5 but not TRPC1 channels. Dev. Biol. 286, 326-37 (2005).

36. Jungnickel, M. K., Marrero, H., Birnbaumer, L., Lémos, J. R. \& Florman, H. M. Trp2 regulates entry of Ca2+ into mouse sperm triggered by egg ZP3. Nat. Cell Biol. 3, 499-502 (2001).

37. Treviño, C. L., Serrano, C. J., Beltrán, C., Felix, R. \& Darszon, A. Identification of mouse trp homologs and lipid rafts from spermatogenic cells and sperm. FEBS Lett. 509, 119-25 (2001).

38. Castellano, L. E. et al. Transient receptor potential (TRPC) channels in human sperm: expression, cellular localization and involvement in the regulation of flagellar motility. FEBS Lett. 541, 69-74 (2003).

39. Kunert-Keil, C., Bisping, F., Krüger, J. \& Brinkmeier, H. Tissue-specific expression of TRP channel genes in the mouse and its variation in three different mouse strains. https://doi.org/10.1186/1471-2164-7-159 (2006).

40. Selvaraj, S. et al. Neurotoxin-induced ER stress in mouse dopaminergic neurons involves downregulation of TRPC1 and inhibition of AKT/mTOR signaling. J. Clin. Invest. 122, 1354-1367 (2012).

41. Li, W., Calfa, G., Larimore, J. \& Pozzo-Miller, L. Activity-dependent BDNF release and TRPC signaling is impaired in hippocampal neurons of Mecp2 mutant mice. Proc. Natl. Acad. Sci. USA 109, 17087-92 (2012).

42. Winn, M. P. et al. A Mutation in the TRPC6 Cation Channel Causes Familial Focal Segmental Glomerulosclerosis. Science (80-.). 308, 1801-1804 (2005).

43. Reiser, J. et al. TRPC6 is a glomerular slit diaphragm-associated channel required for normal renal function. Nat. Genet. 37, 739-744 (2005)

44. Riehle, M. et al. TRPC6 G757D loss-of-function mutation associates with FSGS. J. Am. Soc. Nephrol. 27, 2771-2783 (2016).

45. Wu, X., Eder, P., Chang, B. \& Molkentin, J. D. TRPC channels are necessary mediators of pathologic cardiac hypertrophy. Proc. Natl. Acad. Sci. USA 107, 7000-7005 (2010).

46. Wilkins, B. J. et al. Targeted Disruption of NFATc3, but Not NFATc4, Reveals an Intrinsic Defect in Calcineurin-Mediated Cardiac Hypertrophic Growth. Mol. Cell. Biol. 22, 7603-7613 (2002)

47. Molkentin, J. D. et al. A calcineurin-dependent transcriptional pathway for cardiac hypertrophy. Cell 93, 215-228 (1998).

48. Clause, K. C. \& Barker, T. H. Extracellular matrix signaling in morphogenesis and repair. Curr. Opin. Biotechnol. 24, 830-833 (2013).

49. Frantz, C., Stewart, K. M. \& Weaver, V. M. The extracellular matrix at a glance. J. Cell Sci. 123, 4195-200 (2010).

50. Järveläinen, H., Sainio, A., Koulu, M., Wight, T. N. \& Penttinen, R. Extracellular matrix molecules: potential targets in pharmacotherapy. Pharmacol. Rev. 61, 198-223 (2009).

51. Chin, L., Andersen, J. N. \& Futreal, P. A. Cancer genomics: from discovery science to personalized medicine. Nat. Med. 17, 297-303 (2011).

52. Seo, B. R., DelNero, P. \& Fischbach, C. In vitro models of tumor vessels and matrix: engineering approaches to investigate transport limitations and drug delivery in cancer. Adv. Drug Deliv. Rev. 69-70, 205-216 (2014).

53. Gopal, S. et al. Transmembrane proteoglycans control stretch-activated channels to set cytosolic calcium levels. J. Cell Biol. 210, 1199-1211 (2015)

54. Hakuno, M., Akiyama, M., Shimizu, H., Wheelock, M. J. \& Nishikawa, T. Upregulation of P-cadherin expression in the lesional skin of pemphigus, Hailey-Hailey disease and Darier's disease. J. Cutan. Pathol. 28, 277-81 (2001).

55. Pani, B. et al. Up-regulation of transient receptor potential canonical 1 (TRPC1) following sarco(endo)plasmic reticulum Ca2+ ATPase 2 gene silencing promotes cell survival: a potential role for TRPC1 in Darier's disease. Mol. Biol. Cell 17, 4446-58 (2006).

56. Zhou, S., Matsuyoshi, N., Takeuchi, T., Ohtsuki, Y. \& Miyachi, Y. Reciprocal altered expression of T-cadherin and P-cadherin in psoriasis vulgaris. Br. J. Dermatol. 149, 268-73 (2003).

57. Leuner, K. et al. Reduced TRPC channel expression in psoriatic keratinocytes is associated with impaired differentiation and enhanced proliferation. PLoS One 6, e14716 (2011).

58. Wu, G., Lu, Z.-H., Obukhov, A. G., Nowycky, M. C. \& Ledeen, R. W. Induction of Calcium Influx through TRPC5 Channels by Cross-Linking of GM1 Ganglioside Associated with 51 Integrin Initiates Neurite Outgrowth. https://doi.org/10.1523/ JNEUROSCI.4266-06.2007 (2007).

59. Pedersen, S. F. \& Nilius, B. Transient Receptor Potential Channels in Mechanosensing and Cell Volume Regulation. Methods in enzymology 428, 183-207 (2007).

60. Liu, C. \& Montell, C. Forcing open TRP channels: Mechanical gating as a unifying activation mechanism. Biochem. Biophys. Res. Commun. 460, 22-5(2015).

61. Jemal, I., Soriano, S., Conte, A. L., Morenilla, C. \& Gomis, A. G protein-coupled receptor signalling potentiates the osmomechanical activation of TRPC5 channels. Pflugers Arch. Eur. J. Physiol. 466, 1635-1646 (2014).

62. Wilson, C. \& Dryer, S. E. A mutation in TRPC6 channels abolishes their activation by hypoosmotic stretch but does not affect activation by diacylglycerol or G protein signaling cascades. Am. J. Physiol. Renal Physiol. 306, F1018-25 (2014).

63. Echarri, A. \& Del Pozo, M. A. Caveolae - mechanosensitive membrane invaginations linked to actin filaments. J. Cell Sci. 128, 2747-58 (2015).

64. Litan, A. \& Langhans, S. A. Cancer as a channelopathy: ion channels and pumps in tumor development and progression. Front. Cell. Neurosci. 9, 86 (2015).

65. Wang, G. X. \& Poo, M. Requirement of TRPC channels in netrin-1-induced chemotropic turning of nerve growth cones. Nature 434, 898-904 (2005)

66. Fabian, A. et al. TRPC1 channels regulate directionality of migrating cells. Pflügers Arch. - Eur. J. Physiol. 457, 475-484 (2008).

67. Fabian, A., Bertrand, J., Lindemann, O., Pap, T. \& Schwab, A. Transient receptor potential canonical channel 1 impacts on mechanosignaling during cell migration. Pflugers Arch. 464, 623-30 (2012).

68. Bomben, V. \& Sontheimer, H. Transient Receptor Potential Canonical Channels Are Essential for Chemotactic Migration of Human Malignant Gliomas. J Cell Physiol 31, 1713-1723 (2011). 
69. Bernaldo de Quirós, S. et al. Identification of TRPC6 as a possible candidate target gene within an amplicon at 11q21-q22.2 for migratory capacity in head and neck squamous cell carcinomas. BMC Cancer 13, 116 (2013).

70. Chigurupati, S. et al. Receptor channel TRPC6 is a key mediator of Notch-driven glioblastoma growth and invasiveness. Cancer Res. 70, 418-427 (2010).

71. Henson, E. S. \& Gibson, S. B. Surviving cell death through epidermal growth factor (EGF) signal transduction pathways: implications for cancer therapy. Cell. Signal. 18, 2089-97 (2006).

72. Luo, J., Manning, B. D. \& Cantley, L. C. Targeting the PI3K-Akt pathway in human cancer: Rationale and promise. Cancer Cell 4, 257-262 (2003).

73. Martini, M., De Santis, M. C., Braccini, L., Gulluni, F. \& Hirsch, E. PI3K/AKT signaling pathway and cancer: An updated review. Annals of Medicine 46, 372-383 (2014).

74. Shen, B. et al. cAMP Activates TRPC6 Channels via the Phosphatidylinositol 3-Kinase (PI3K)-Protein Kinase B (PKB)-Mitogenactivated Protein Kinase Kinase (MEK)-ERK1/2 Signaling Pathway. J. Biol. Chem. 286, 19439-19445 (2011).

75. Zhou, L.-F. et al. TRPC6 contributes to LPS-induced inflammation through ERK1/2 and p38 pathways in bronchial epithelial cells. Am J Physiol Cell Physiol 314, 278-288 (2018).

76. Shi, J., Ju, M., Large, W. A., Albert, A. P. \& Albert, A. P. Pharmacological profile of phosphatidylinositol 3-kinases and related phosphatidylinositols mediating endothelin A receptor-operated native TRPC channels in rabbit coronary artery myocytes Keywords canonical transient receptor potential; endothelin; phosphatidylinositol 3-kinase; phosphatidylinositol; vascular smooth muscle. Br. J. Pharmacol. 166, 2161 (2012).

77. Xie, J. et al. Cardioprotection by Klotho through downregulation of TRPC6 channels in the mouse heart HHS Public Access Author manuscript. Nat Commun 3, 1238 (2012).

78. Monet, M., Francoeur, N. \& Boulay, G. Involvement of Phosphoinositide 3-Kinase and PTEN Protein in Mechanism of Activation of TRPC6 Protein in Vascular Smooth Muscle Cells. J. Biol. Chem. 287, 17672-17681 (2012).

79. Bezzerides, V. J., Ramsey, I. S., Kotecha, S., Greka, A. \& Clapham, D. E. Rapid vesicular translocation and insertion of TRP channels. Nat. Cell Biol. 6, 709-720 (2004).

80. Zhang, K. et al. Regulation of TRPC1 channel by endothelin-1 in human atrial myocytes. Hear. Rhythm. https://doi.org/10.1016/j. hrthm.2019.04.003 (2019)

81. Heiser, J. H. et al. TRPC6 channel-mediated neurite outgrowth in PC12 cells and hippocampal neurons involves activation of RAS/ MEK/ERK, PI3K, and CAMKIV signaling. J. Neurochem. 127, 303-313 (2013).

82. Kim, J.-E. \& Kang, T.-C. TRPC3- and ETB receptor-mediated PI3K/AKT activation induces vasogenic edema formation following status epilepticus. Brain Res. 1672, 58-64 (2017).

83. Yang, L.-P. et al. Acute Treatment with a Novel TRPC4/C5 Channel Inhibitor Produces Antidepressant and Anxiolytic-Like Effects in Mice. https://doi.org/10.1371/journal.pone.0136255 (2015).

84. Zanou, N. et al. Trpc1 Ion Channel Modulates Phosphatidylinositol 3-Kinase/Akt Pathway during Myoblast Differentiation and Muscle Regeneration *. https://doi.org/10.1074/jbc.M112.341784 (2012).

85. Azimi, I. et al. TRPC1 is a differential regulator of hypoxia-mediated events and Akt signalling in PTEN-deficient breast cancer cells. J. Cell Sci. 130, 2292-2305 (2017).

86. Bell-Pedersen, D. et al. Circadian rhythms from multiple oscillators: Lessons from diverse organisms. Nature Reviews Genetics 6, 544-556 (2005).

87. Krzeptowski, W. et al. Circadian Plasticity in the Brain of Insects and Rodents. https://doi.org/10.3389/fncir.2018.00032 (2018).

88. Song, C. et al. Insights into the Role of Circadian Rhythms in Bone Metabolism: A Promising Intervention Target? https://doi. org/10.1155/2018/9156478 (2018)

89. Bellemer, A. Thermotaxis, circadian rhythms, and TRP channels in Drosophila. Temperature., https://doi.org/10.1080/23328940.2 $015.1004972(2015)$.

90. Ripperger, J. A. \& Schibler, U. Circadian regulation of gene expression in animals. Curr. Opin. Cell Biol. 13, 357-362 (2001).

91. Paria, B. C. et al. Tumor necrosis factor-alpha-induced TRPC1 expression amplifies store-operated Ca2+ influx and endothelial permeability. Am. J. Physiol. Lung Cell. Mol. Physiol. 287, L1303-13 (2004).

92. Py, B. F. et al. Caspase-11 controls interleukin-1 $\beta$ release through degradation of TRPC1. Cell Rep. 6, 1122-1128 (2014).

93. Medic, N. et al. Knockout of the Trpc1 gene reveals that TRPC1 can promote recovery from anaphylaxis by negatively regulating mast cell TNF- $\alpha$ production. Cell Calcium 53, 315-26 (2013).

94. Yu, Q. \& Li, M. Effects of transient receptor potential canonical 1 (TRPC1) on the mechanical stretch-induced expression of airway remodeling-associated factors in human bronchial epithelioid cells. J. Biomech. 51, 89-96 (2017).

95. Zhou, X. et al. Transient Receptor Potential Channel 1 Deficiency Impairs Host Defense and Proinflammatory Responses to Bacterial Infection by Regulating Protein Kinase Co Signaling. Mol. Cell. Biol. 35, 2729-39 (2015).

96. Stewart, T. A., Azimi, I., Thompson, E. W., Roberts-Thomson, S. J. \& Monteith, G. R. A role for calcium in the regulation of ATPbinding cassette, sub-family $\mathrm{C}$, member 3 (ABCC3) gene expression in a model of epidermal growth factor-mediated breast cancer epithelial-mesenchymal transition. Biochem. Biophys. Res. Commun. 458, 509-514 (2015).

97. Ge, P. et al. TRPC1/3/6 inhibition attenuates the TGF- 31 -induced epithelial-mesenchymal transition in gastric cancer via the Ras/ Raf1/ERK signaling pathway. Cell Biol. Int. 42, 975-984 (2018).

98. Solanki, S., Dube, P. R., Birnbaumer, L. \& Vazquez, G. Reduced Necrosis and Content of Apoptotic M1 Macrophages in Advanced Atherosclerotic Plaques of Mice With Macrophage-Specific Loss of Trpc3. Sci. Rep. 7, 42526 (2017).

99. Feng, M., Xu, D. \& Wang, L. miR-26a inhibits atherosclerosis progression by targeting TRPC3. Cell Biosci. 8, 4 (2018).

100. Dietrich, A. Transient Receptor Potential (TRP) Channels in Health and Disease. Cells 8, 413 (2019).

101. Mizoguchi, Y. \& Monji, A. TRPC Channels and Brain Inflammation. In Advances in experimental medicine and biology 976, 111-121 (2017).

102. Hang, P. et al. Brain-derived neurotrophic factor regulates TRPC $3 / 6$ channels and protects against myocardial infarction in rodents. Int. J. Biol. Sci. 11, 536-45 (2015).

103. Mizoguchi, Y. et al. Brain-derived neurotrophic factor (BDNF) induces sustained intracellular $\mathrm{Ca} 2+$ elevation through the upregulation of surface transient receptor potential 3 (TRPC3) channels in rodent microglia. J. Biol. Chem. 289, 18549-55 (2014).

104. Thilo, F., Scholze, A., Liu, D. Y., Zidek, W. \& Tepel, M. Association of transient receptor potential canonical type 3 (TRPC3) channel transcripts with proinflammatory cytokines. Arch. Biochem. Biophys. 471, 57-62 (2008).

105. Alawi, K. M. et al. Transient receptor potential canonical 5 (TRPC5) protects against pain and vascular inflammation in arthritis and joint inflammation. Ann. Rheum. Dis. 76, 252-260 (2017).

106. Wang, J. et al. Cross-linking of GM1 ganglioside by galectin-1 mediates regulatory T cell activity involving TRPC5 channel activation: possible role in suppressing experimental autoimmune encephalomyelitis. J. Immunol. 182, 4036-45 (2009).

107. Ramirez, G. A. et al. cells Ion Channels and Transporters in Inflammation: Special Focus on TRP Channels and TRPC6. https:// doi.org/10.3390/cells7070070.

108. Singh, I. et al. Galphaq-TRPC6-mediated $\mathrm{Ca} 2+$ entry induces RhoA activation and resultant endothelial cell shape change in response to thrombin. J. Biol. Chem. 282, 7833-43 (2007).

109. Kini, V., Chavez, A. \& Mehta, D. A new role for PTEN in regulating transient receptor potential canonical channel 6-mediated Ca2+ entry, endothelial permeability, and angiogenesis. J. Biol. Chem. 285, 33082-91 (2010). 
110. Damann, N., Owsianik, G., Li, S., Poll, C. \& Nilius, B. The calcium-conducting ion channel transient receptor potential canonical 6 is involved in macrophage inflammatory protein-2-induced migration of mouse neutrophils. Acta Physiol. (Oxf). 195, 3-11 (2009).

111. Lindemann, O. et al. TRPC6 regulates CXCR2-mediated chemotaxis of murine neutrophils. J. Immunol. 190, 5496-505 (2013).

112. Chen, W. et al. Atrial natriuretic peptide-mediated inhibition of microcirculatory endothelial Ca2+ and permeability response to histamine involves cGMP-dependent protein kinase I and TRPC6 channels. Arterioscler. Thromb. Vasc. Biol. 33, 2121-9 (2013).

113. Tauseef, M. et al. TLR4 activation of TRPC6-dependent calcium signaling mediates endotoxin-induced lung vascular permeability and inflammation. J. Exp. Med. 209, 1953-68 (2012).

114. Kurahara, L. H. et al. Intestinal Myofibroblast TRPC6 Channel May Contribute to Stenotic Fibrosis in Crohn's Disease. Inflamm. Bowel Dis. 21, 496-506 (2015).

115. Xu, J. et al. The NCX1/TRPC6 Complex Mediates TGFß-Driven Migration and Invasion of Human Hepatocellular Carcinoma Cells. Cancer Res. 78, 2564-2576 (2018).

116. Liu, X. et al. Attenuation of store-operated Ca2+ current impairs salivary gland fluid secretion in TRPC1(-/-) mice. Proc. Natl. Acad. Sci. USA 104, 17542-7 (2007).

117. Ong, E.-C. et al. A TRPC1 Protein-dependent Pathway Regulates Osteoclast Formation and Function. J. Biol. Chem. 288, 22219-22232 (2013)

118. He, K. et al. Movement deficits and neuronal loss in basal ganglia in TRPC1 deficient mice. Oncotarget 7, 69337-69346 (2016).

119. Hasen, N. S. \& Gammie, S. C. Trpc2-deficient lactating mice exhibit altered brain and behavioral responses to bedding stimuli. Behav. Brain Res. 217, 347-353 (2011).

120. Stowers, L., Holy, T. E., Meister, M., Dulac, C. \& Koentges, G. Loss of Sex Discrimination and Male-Male Aggression in Mice Deficient for TRP2. Science (80-.). 295, 1493-1500 (2002).

121. Hartmann, J. et al. TRPC3 Channels Are Required for Synaptic Transmission and Motor Coordination. Neuron 59, 392-398 (2008).

122. Hasna, J., Abi Nahed, R., Sergent, F., Alfaidy, N. \& Bouron, A. The Deletion of TRPC6 Channels Perturbs Iron and Zinc Homeostasis and Pregnancy Outcome in Mice. Cell. Physiol. Biochem. 52, 455-467 (2019).

123. Freichel, M. et al. Lack of an endothelial store-operated Ca2+ current impairs agonist-dependent vasorelaxation in TRP4-/mice. Nat. Cell Biol. 3, 121-127 (2001).

124. Phelan, K. D. et al. Canonical transient receptor channel 5 (TRPC5) and TRPC1/4 contribute to seizure and excitotoxicity by distinct cellular mechanisms. Mol. Pharmacol. 83, 429-38 (2013).

125. Dietrich, A. et al. Increased Vascular Smooth Muscle Contractility in TRPC6-/- Mice. Mol. Cell. Biol. 25, 6980-6989 (2005).

126. Perez-Leighton, C. E., Schmidt, T. M., Abramowitz, J., Birnbaumer, L. \& Kofuji, P. Intrinsic phototransduction persists in melanopsin-expressing ganglion cells lacking diacylglycerol-sensitive TRPC subunits. Eur. J. Neurosci. 33, 856-67 (2011).

127. Hochberg, Y. \& Benjamini, Y. More powerful procedures for multiple significance testing. Stat. Med. 9, 811-818 (1990).

128. Nowotarski, S. L., Woster, P. M. \& Casero, R. A. Polyamines and cancer: implications for chemotherapy and chemoprevention. Expert Rev. Mol. Med. 15, e3 (2013).

\section{Acknowledgements}

This research was supported in part by the Intramural Research Program of the NIH (Project Z01-ES-101684 to LB). We thanks Drs. Adam Burkholder and David Fargo, from the Office of the Scientific Director (NIEHS) for building the Table with the lists of Differentially Expressed Genes (Supplementary Table).

\section{Author contributions}

K.F., S.S. performed experiments; L.B., designed experiments; M.F. provided reagents, K.F., S.S. and M.F. and L.B. wrote the paper.

\section{Competing interests}

The authors declare no competing interests.

\section{Additional information}

Supplementary information is available for this paper at https://doi.org/10.1038/s41598-020-61177-x.

Correspondence and requests for materials should be addressed to L.B.

Reprints and permissions information is available at www.nature.com/reprints.

Publisher's note Springer Nature remains neutral with regard to jurisdictional claims in published maps and institutional affiliations.

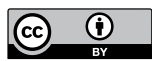

Open Access This article is licensed under a Creative Commons Attribution 4.0 International License, which permits use, sharing, adaptation, distribution and reproduction in any medium or format, as long as you give appropriate credit to the original author(s) and the source, provide a link to the Creative Commons license, and indicate if changes were made. The images or other third party material in this article are included in the article's Creative Commons license, unless indicated otherwise in a credit line to the material. If material is not included in the article's Creative Commons license and your intended use is not permitted by statutory regulation or exceeds the permitted use, you will need to obtain permission directly from the copyright holder. To view a copy of this license, visit http://creativecommons.org/licenses/by/4.0/.

This is a U.S. Government work and not under copyright protection in the US; foreign copyright protection may apply 2020 